Review

\title{
Ferroptosis in Cancer Progression: Role of Noncoding RNAs
}

\author{
Ying-Bing Zuo ${ }^{1,2^{*}}$, Yin-Feng Zhang ${ }^{2 * \varpi}$, Rui Zhang ${ }^{1}$, Jia-Wei Tian ${ }^{3}$, Xiao-Bing Lv ${ }^{1}$, Rong Li1 ${ }^{1}$, Shu-Ping Li ${ }^{4}$, \\ Meng-Die Cheng ${ }^{1,2}$, Jing Shan ${ }^{2,5,6}$, Zheng Zhao ${ }^{1,2}$, Hui Xin ${ }^{1 凶}$ \\ 1. Department of Cardiology, The Affiliated Hospital of Qingdao University, Qingdao University, Qingdao 266000, Shandong, P.R. China. \\ 2. Institute for Translational Medicine, The Affiliated Hospital of Qingdao University, College of Medicine, Qingdao University, Deng Zhou Road 38, \\ Qingdao 266021, Shandong, P.R. China. \\ 3. Department of Emergency Internal Medicine, The Affiliated Hospital of Qingdao University, Qingdao 266000, Shandong, P.R. China. \\ 4. Department of Cardiology, The Affiliated Qingdao Third People's Hospital of Qingdao University, Qingdao University, Qingdao 266041, Shandong, P.R. \\ China. \\ 5. Key Laboratory of Environmental Factors and Chronic Disease Control, Yinchuan 750004, Ningxia, P.R. China \\ 6. School of Public Health and Management, Ningxia Medical University, Yinchuan 750004, Ningxia, P.R. China. \\ "These authors contributed equally. \\ $\triangle$ Corresponding authors: Yin-Feng Zhang, ORCID: https://orcid.org/0000-0003-2457-7712, Email address: zhangyinfeng@qdu.edu.cn; Hui Xin, Email \\ address: xinhuiqy@163.com
}

( ) The author(s). This is an open access article distributed under the terms of the Creative Commons Attribution License (https://creativecommons.org/licenses/by/4.0/). See http://ivyspring.com/terms for full terms and conditions.

Received: 2021.09.08; Accepted: 2022.01.16; Published: 2022.02.14

\begin{abstract}
Ferroptosis is a novel form of programmed cell death, and it is characterized by iron-dependent oxidative damage, lipid peroxidation and reactive oxygen species accumulation. Notable studies have revealed that ferroptosis plays vital roles in tumor occurrence and that abundant ferroptosis in cells can inhibit tumor progression. Recently, some noncoding RNAs (ncRNAs), particularly microRNAs, long noncoding RNAs, and circular RNAs, have been shown to be involved in biological processes of ferroptosis, thus affecting cancer growth. However, the definite regulatory mechanism of this phenomenon is still unclear. To clarify this issue, increasing studies have focused on the regulatory roles of ncRNAs in the initiation and development of ferroptosis and the role of ferroptosis in progression of various cancers, such as lung, liver, and breast cancers. In this review, we systematically summarized the relationship between ferroptosis-associated ncRNAs and cancer progression. Moreover, additional evidence is needed to identify the role of ferroptosis-related ncRNAs in cancer progression. This review will help us to understand the roles of ncRNAs in ferroptosis and cancer progression and may provide new ideas for exploring novel diagnostic and therapeutic biomarkers for cancer in the future.
\end{abstract}

Key words: Ferroptosis; microRNAs; long noncoding RNAs; circular RNAs; Cell death

\section{Introduction}

\subsection{Mechanism of ferroptosis}

Programmed cell death (PCD) is important for the balance between the progression of diseases and human health [1]. Ferroptosis, a novel coined form of PCD discovered in 2012 [2], is different from apoptosis, necroptosis, pyroptosis and autophagy [3]. Many studies have revealed that ferroptosis is a specific oxidative and iron-dependent form of PCD caused by abnormal iron metabolism and lethal lipid peroxidation [4,5]. Moreover, some studies have demonstrated that autophagy plays a crucial role in ferroptosis, especially autophagic degradation of ferroptosis-related proteins, such as ferritinophagy, lipophagy, clockophagy and chaperone-mediated autophagy [6,7]. Recently, an increasing number of studies have focused on the role of ferroptosis in various diseases $[8,9]$, especially liver, lung, and gastrointestinal cancers [10]. By exploring the molecular mechanism related to the regulation of ferroptosis more deeply, the relationship between ferroptosis and cancer progression will be better understood. 


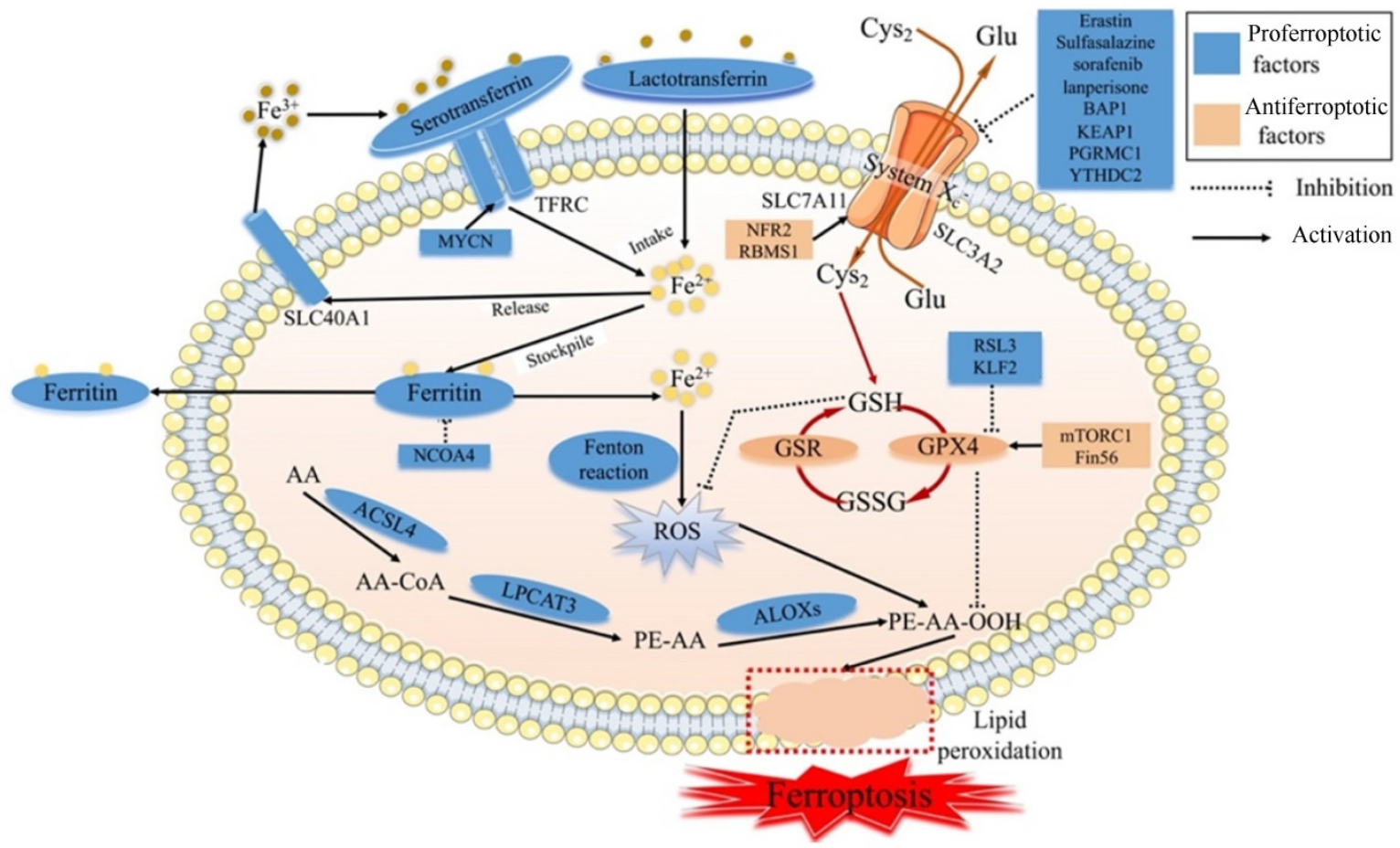

Fig. 1. The molecular mechanism of ferroptosis. Cys2: Cysteine; Glu: Glutamate; SLC7A11: Solute carrier family 7 membrane 11; SLC3A2: Solute carrier family 3 membrane 2; SLC40A1: Solute carrier family 40 membrane 1; TFRC: Transferrin receptor; GSH: Reduced glutathione; GPX4: Glutathione peroxidase 4; GSR: Glutathione reductase; GSSG: Oxidized Glutathione; ROS: Reactive oxygen species; AA: Arachidonic acid; ACSL4: Acyl-CoA synthetase long-chain family member 4; LPCAT3: lysophophatidylcholne acyltransferase 3; ALOXs: Lipoxygenases

Ferroptosis is regulated by specific signal transduction pathways through iron accumulation, lipid peroxidation and cellular membrane destruction, and ferroptosis can be modulated by drugs or genetic interventions [11] (Fig. 1). The main mechanism of ferroptosis involves regulating homeostasis between oxidative and antioxidant systems [12].

\subsubsection{Iron in ferroptosis}

Iron accumulation plays a critical role in producing ROS via the Fenton reaction and enzyme activity in terms of lipid peroxidation. Although iron is essential in physiological processes, excessive iron is pernicious and can trigger ferroptosis. Ferroptosis is strictly regulated by modulators related to iron metabolism processes, such as iron intake, stockpile, usage, and release [13]. Serotransferrin- or lactotransferrin-associated iron intake promotes ferroptosis through the transferrin receptor (TFRC) $[14,15]$. Furthermore, oncogenic MYCN could induce iron accumulation by increasing the expression of TFRC [16]. The cargo receptor NCOA4 could activate autophagy to degrade ferritin, a process called ferritinophagy, leading to the promotion of ferroptosis [17]. In contrast, solute carrier family 40 membrane 1 (SLC40A1)-associated iron release inhibits ferroptosis [18]. Along with the degradation of ferritin, the level of intracellular iron is high, leading to ferroptosis [19], whereas ferritin efflux inhibits ferroptosis. Evidence revealed that the transcription factor $\mathrm{BACH} 1$ could reduce iron accumulation by upregulating the translation of ferritin genes (Fth1 and Flt1) and the ferroportin gene (SLC40A1) and inhibiting ferroptosis [20]. Some mitochondrial proteins associated with the usage of iron negatively regulate ferroptosis, such as NFS1 [21], ISCU[22], CISD1 [23] and CISD2 [24]. Moreover, the iron accumulation could be regulated by some signal transduction pathways. Nuclear protein 1 (NUPR1) , a transcriptional regulator, blocks cell ferroptosis and decreases iron accumulation by increasing the production of the iron transporter LCN2 [25]. Recent studies have revealed that iron chelators and antioxidants can inhibit ferroptosis [26-28].

\subsubsection{Lipid peroxidation in ferroptosis}

Lipid peroxidation leading to cell membrane destruction is the central intermediate link of ferroptosis. The molecular mechanism of lipid peroxidation is that the inhibition of system $\mathrm{XC}^{-}$or glutathione peroxidase 4 (GPX4) leads to decreased production of reduced GSH [14]. The glutamate $(\mathrm{Glu}) /$ cysteine $\left(\mathrm{Cys}_{2}\right)$ antiporter of system $\mathrm{Xc}_{\mathrm{c}^{-}}$is composed of solute carrier family 3 membrane 2 (SLC3A2) and solute carrier family 7 membrane 11 (SLC7A11) and can import extracellular Cys 2 into cells 
and exchange intracellular Glu. Moveover, some evidence has revealed that the cargo receptor SQSTM1/p62 can induce the autophagic degradation of ARNTL, known as clockophagy, and promote lipid peroxidation and ferroptosis via the EGLN2/HIF1A pathway $[29,30]$.

\section{Inhibiting system $\mathrm{Xc}^{-}$}

System $\mathrm{XC}^{-}$, which involves GSH, is one of the main antioxidant defenses in our body. When system $\mathrm{Xc}^{-}$is inhibited by erastin, $\mathrm{Cys}_{2}$ cannot influx cells, resulting in a decrease in the amount of $\mathrm{Cys}_{2}$. Due to the important role of $\mathrm{Cys}_{2}$ in the process of $\mathrm{GSH}$ biosynthesis, $\mathrm{Cys}_{2}$ deficiency can reduce the level of GSH. Then, the exhaustion of GSH can reduce the expression and activity of GPX4 [31,32]. Meanwhile, GPX4 can catalyze GSH to GSSG, and then toxic peroxides can be reduced to nontoxic hydroxyl compounds to protect the structure and function of the cell membrane from interference and destruction by peroxides [33]. Along with the destruction of the redox balance in cells, the accumulation of ROS can induce cell membrane breakage and cell death [34]. The imbalance of system $\mathrm{Xc}_{\mathrm{c}^{-}}$is one of the main biochemical features of ferroptosis, and the regulation of ferroptosis is the primary way to control its occurrence.

Several studies uncovered that ferroptosis could be induced by inhibiting system $\mathrm{X}_{\mathrm{C}^{-}}$via certain compounds, such as erastin, sulfasalazine [4], sorafenib, and lanperisone [35]. Moveover, studies have identified that some genes could regulate the catalytic subunit of system $\mathrm{Xc}_{\mathrm{c}^{-}}$. These genes regulate the transcription or translation of subunits of system

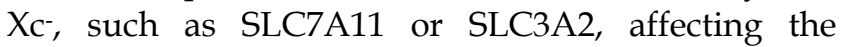
biological processes of the ferroptosis. The tumor suppressor BRCA1-related protein1 (BAP1) could inhibit the SLC7A11 expression and then lead to elevated lipid peroxidation and ferroptosis [36]. In some cancers, Kelch-like ECH-associated protein 1 (KEAP1) depresses the translation of SLC7A11 and reduces the exchange of Glu/Cys2, otherwise NF E2-related factor 2 (NRF2) plays the opposite role in SLC7A11 [37]. Hence, the Nrf2/Keap1 pathway promotes the translation of SLC7A11, leading to diminished ferroptosis [38]. In lung cancer, the RNA-binding protein RBMS1 could promote the levels of SLC7A11 and increase the production of $\mathrm{GSH}$, resulting in the inhibition of ferroptosis in cancer cells [39]. In terms of the relationship between autophagy and lipid peroxidation, progesterone receptor membrane component 1 (PGRMC1) suppresses SLC7A11 via autophagic degradation of lipids, known as lipophagy, and induces ferroptosis in paclitaxel-tolerant persister cancer cells [40]. P53, a well-known tumor suppressor gene, can inhibit the expression of SLC7A11, leading to lipid peroxidation and ferroptosis [41]. YTHDC2, an $\mathrm{m}^{6} \mathrm{~A}$ reader identified in 2017, suppresses the expression of SLC3A2 by inhibiting HOXA13, a transcription factor of SLC3A2 expression, to trigger system $\mathrm{XC}^{-}$ -dependent ferroptosis [42].

\section{Inhibiting GPX4}

GPX4, another main antioxidant defense, can directly reduce cell membrane phospholipid hydroperoxide to hydroxyphospholipid, taking advantage of GSH as a substrate, resulting in the suppression of ferroptosis in cancer cells [43]. The inhibition of GPX4 by RSL3 induces ferroptosis [44]. Recently, many studies have revealed that some factors regulate the generation process of GPX4. Boyi Gan and his team discovered that rapamycin complex 1 (mTORC1), a foremost regulator of cell growth and metabolism, increased the production of GPX4 protein and reduced the production of lipid peroxidation of the cell membrane [45-47]. Moreover, Fin56, a ferroptosis inducer, synergized with Torin 2, promoting GPX4 translation and triggering ferroptosis in bladder cancer cells [48]. In other types of cancer, KLF2 reduces the transcriptional repression of GPX4 to prevent the lipid peroxidation and inhibit ferroptosis in clear cell renal cell carcinoma [49]. Interestingly, erastin activation of ferroptosis increased the production of lysosome-associated membrane protein $2 \mathrm{a}$ and induced chaperonemediated autophagy, which in turn increased the degradation of GPX4 resulting in reduced ferroptosis [50].

Genetic depletion of GPX4 causes lipid peroxidation and then induces ferroptosis in cancer cells or tissues [51]. In the process of lipid metabolism, arachidonic acid (AA) can produce AA-CoA through acyl-CoA synthetase long-chain family member 4 (ACSL4), and AA-CoA is esterified by lysophophatidylcholine acyltransferase 3 (LPCAT3) to produce phosphatidyl-(PE)-AA [52]. PE-AA is oxidized to PE-AA-OOH by lipoxygenases (LOXs), leading to degradation of the cell membrane [53]. Cytotoxic PE-AA-OOH is usually reduced to noncytotoxic PE-AA-OH, protecting cells from oxidative damage via GPX4. However, when GPX4 is deficient or inactivated, PE-AA-OOH cannot be reduced and then induces ferroptosis [53]. Overall, GPX4 systems are also crucial for the occurrence of ferroptosis.

\subsubsection{ROS in ferroptosis}

In the process of lipid peroxidation, the lethal accumulation of lipid ROS can destroy the cell 
membrane, leading to ferroptosis [54]. ROS are produced in two main ways: the Fenton reaction with $\mathrm{Fe}^{2+}$ and lipid peroxidation. When the two antioxidant defenses, GSH and GPX4 systems, are impaired, the accumulation of toxic ROS will occur and then induce cell death. Some studies have revealed that erastin causes the production of ROS in some cell lines [4,55]. Cells treated with RSL3 revealed elevated lipid ROS during ferroptosis in the absence of GSH depletion. With prolonged erastin and RSL3 treatment times, ROS can begin to accumulate and induce cancer cell ferroptosis [4] Furthermore, ROS are generated from the TCA cycle of mitochondrial metabolism.

\subsection{Inhibition of cancer progression by ferroptosis}

Many studies have indicated that ferroptosis plays a crucial role in the regulation of the pathological process of cancer [56,57]. Some studies have revealed that superabundant ferroptosis of cancer cells can inhibit tumor progression. Several anticancer drugs could inhibit ferroptosis-related molecules and channels to induce ferroptosis in cancer cells, such as GPX4 and system $\mathrm{Xc}^{-}$, and then inhibit cancer growth $[5,58]$. It was found that the ferroptosis inducer erastin could increase the chemotherapeutic effect of some chemotherapeutics, such as cisplatin [59], cytosine arabinoside and doxorubicin [60], by inducing ferroptosis. Similarly, inactivation of dihydroorotate dehydrogenase led to a large amount of mitochondrial lipid peroxidation and induced ferroptosis in cancer cells [61]. Moreover, radiotherapy could cause cancer cells to produce lipid ROS and result in the lethal accumulation of lipid peroxides to induce ferroptosis [62]. Hence, the induction of ferroptosis may become a promising strategy to treat cancer. Next, we will discuss the relationship between ncRNAs and ferroptosis.

\subsection{Function of ncRNAs in ferroptosis}

Ferroptosis is related to the prognosis of many types of cancer, but we know little of the mechanism of the ferroptosis in cancer, especially regarding the role of ferroptosis-related ncRNAs in cancer. Ferroptosis is tightly related to noncoding RNAs (ncRNAs) and cancer [63]. NcRNAs, including microRNAs (miRNAs), long noncoding RNAs (lncRNAs) (Fig. 2), and circular RNAs (circRNAs) (Fig. 3), are involved in the underlying regulatory mechanism of ferroptosis, including mitochondrial-related proteins, iron metabolism, glutathione metabolism, and lipid peroxidation $[64,65]$.

In terms of regulating ferroptosis-related genes, some studies have demonstrated that many ncRNAs play vital roles in regulating the expression of the ferroptosis-related genes [66]. Some ncRNAs regulate ferroptosis in cancer cells by affecting the protein level of ferroptosis-associated genes, such as FSP1 [67], EIF4A1 [68], GABPB1 [69], GDPD5 [70], and CCL5 [71]. A number of ncRNAs could affect both the mRNA and protein levels of ferroptosis-associated genes, such as NRF2 [72], STAT3 [73], ATF4 [74], AURKA [75], and ITGB8 [76]. What's more, several miRNAs could participate at the mRNA or protein level via m6A modification or epigenetic regulation of these genes, such as FSP1 [67] and AURKA [75]. Some circRNAs could regulate these genes by sponging miRNAs at the mRNA and protein levels. Moreover, lncRNAs could regulate the expression of ferroptosis-related proteins by affecting p53 at the transcriptional level. For example, lncRNA P53RRA could promote the recruitment of p53 by interacting with G3BP1 and then regulate the function of ferroptosis-associated metabolic genes [77]. Moreover, some ncRNAs could induce ferroptosis by regulating iron metabolism. Some studies have revealed that many ncRNAs increase the content of cellular iron [72,77-79]. Apart from this, several ncRNAs could decrease the accumulation of iron [80-82]. Moreover, miR-7-5p plays a vital role in downregulating mitoferrin, reducing $\mathrm{Fe}^{2+}$ [83], and inhibiting the ferroptosis. In addition, many ncRNAs could affect ferroptosis via ROS metabolism by the Fenton reaction $[69,73,79,80]$. However, future studies need to pay more attention to the molecular mechanism of the relationship between ferroptosis-related ncRNAs and iron or ROS metabolism.

Regarding lipid peroxidation, ncRNAs regulate the subunits of system $\mathrm{Xc}^{-}$and GPX4, and several ncRNAs play important roles in the process of lipid metabolism. Many ncRNAs regulated the protein level of SLC7A11 [78,84-87]. Moreover, some circRNAs regulated SLC7A11 by sponging miRNAs. Furthermore, several studies revealed that ncRNAs could increase the levels of SLC7A11 by promoting the recruitment of LSH to the promoter of SLC7A11 [79]. In the aspect of GPX4, many studies have demonstrated that ncRNAs focus on regulating the protein production of GPX4 [88-92]. A few studies have revealed that circRNAs can upregulate the levels of GPX4 mRNA [91]. Several studies have demonstrated that lncRNAs and circRNAs can regulate the GPX4 by interacting with miRNAs. For instance, lncRNA PVT1 induced the expression of GPX4 by inhibiting the translation of miR-214-3p [90]. In addition, has_circ_0048179 increased the level of GPX4 by sponging miR-188-3p [93]. Regarding the uptake of Gln, miRNAs could regulate the expression of the Glu metabolism-related proteins and affect the ferroptosis of cells. For example, miR-103a-3p 
inhibited the expression of glutaminase 2 (GLS2) and suppressed the glutamine transformation to glutamate [94]. miR-9 could inhibit the mRNA and protein levels of glutamic-oxaloacetic transaminase 1 (GOT1) and inhibit Glu metabolism [95]. In addition, miR-137 inhibited glutaminolysis by suppressing the glutamine transporter solute carrier family 1 membrane 5 (SLC1A5) [81]. Moreover, several studies have revealed that ncRNAs can induce lipid peroxidation by regulating the translation of the fundamental enzymes for the biosynthesis of unsaturated phospholipids, such as ACSL4 [96,97], ALOX15 [80], and ALOXE3 [98]. In summary, ncRNAs play crucial roles in many biological processes of ferroptosis occurrence and development.

Recently, an increasing amount of evidence has demonstrated that ncRNAs play an important regulatory role in cancer progression via the ferroptosis pathway $[3,64]$ and might become new diagnostic markers or therapeutic targets of cancers. Hence, this review focuses on summarizing the regulatory role of ncRNAs in cancer progression via ferroptosis of cancer cells (Table 1). Moreover, there may be some obstacles to hindering the exploration of ferroptosis-related ncRNAs in cancer therapy or diagnosis. We believe that a deep understanding of the interactions between ncRNAs and ferroptosis may be conducive to solving these obstacles and improving the strategy of cancer therapy or diagnosis.

\section{Role of ncRNAs in cancer progression via the ferroptosis pathway}

\subsection{Lung cancer}

Lung cancer is the leading cause of cancer-associated deaths, with approximately 1.8 million deaths $(18 \%)$, and it is the most common cancer in men worldwide, with an estimated 1.44 million new cases each year (14.3\%) [99]. Lung cancer is divided into small cell lung cancer (SCLC) and non-small-cell lung cancer (NSCLC). NSCLC, including lung adenocarcinoma (ADC) and squamous cell carcinoma (SCC), is the most frequently occurring cancer among lung cancers [100]. Although there are many therapeutic strategies for lung cancer therapy, such as surgical sectioning, chemotherapy, and radiotherapy, there is still a lack of a definite understanding of the pathogenesis of lung cancer. Thus, identifying novel diagnostic markers and therapeutic strategies to inhibit the progression of lung cancer is a great challenge. However, second-generation sequencing technology has been widely used to provide a very effective way to study disease-related genes and ncRNAs in recent years $[101,102]$. To a certain extent, this technology will help us to predict ferroptosis-related ncRNAs.

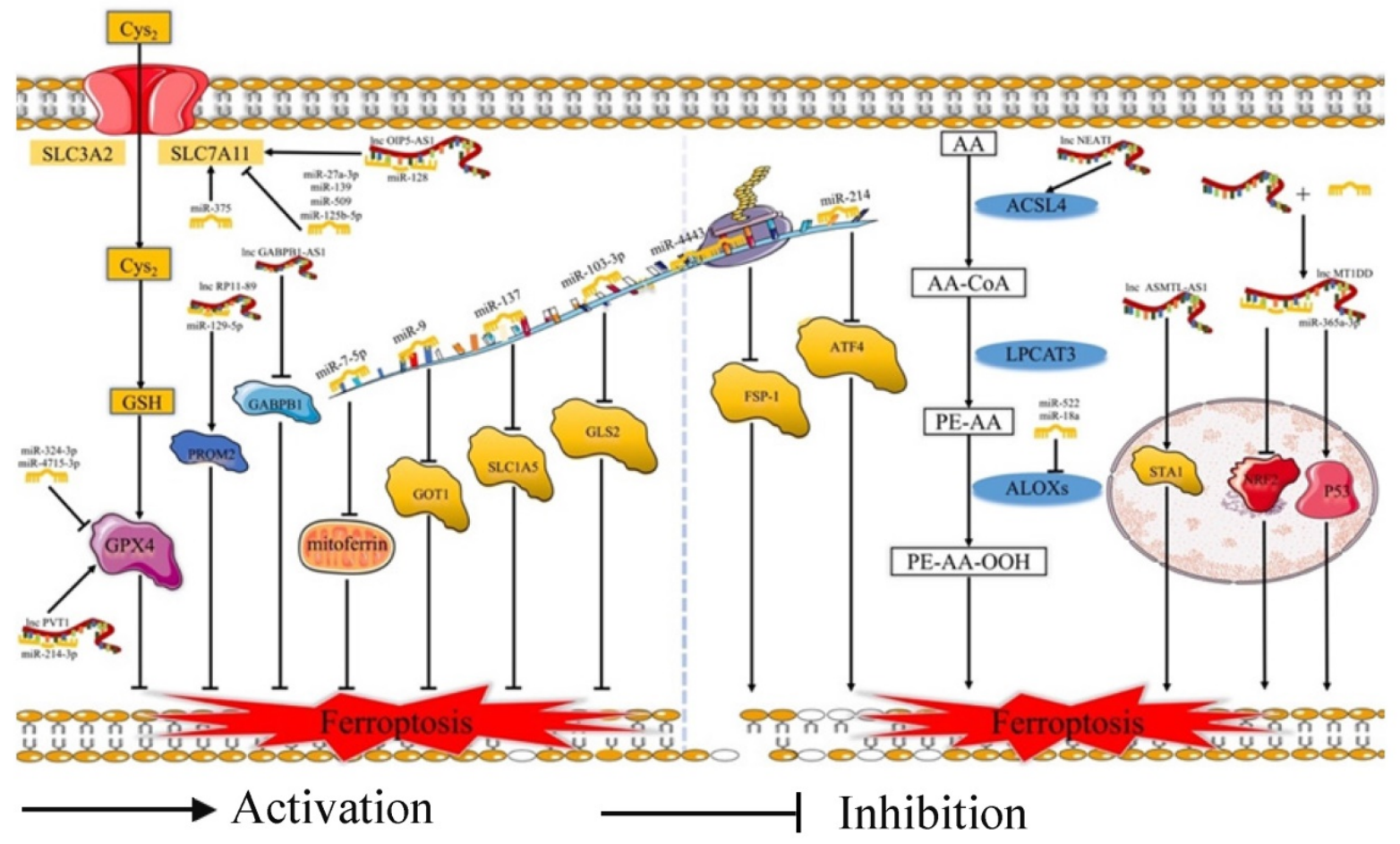

Fig. 2. MiRNAs and IncRNAs play regulatory role in ferroptosis. Cys2: Cysteine; GSH: Reduced glutathione; SLC3A2: Solute carrier family 3 membrane 2; SLC7A1 1: Solute carrier family 7 membrane 11; GPX4: Glutathione peroxidase 4; GOT1: Glutamic-oxaloacetic transaminase 1; SLC1A5: Solute carrier family 1 membrane 5; GLS2: Glutaminase 2; FSP-1: Ferroptosis suppressor protein 1; ATF4: Activating transcription factor 4; AA: Arachidonic acid; ACSL4: Acyl-CoA synthetase long-chain family member 4; LPCAT3: lysophophatidylcholne acyltransferase 3; ALOXs: Lipoxygenases 


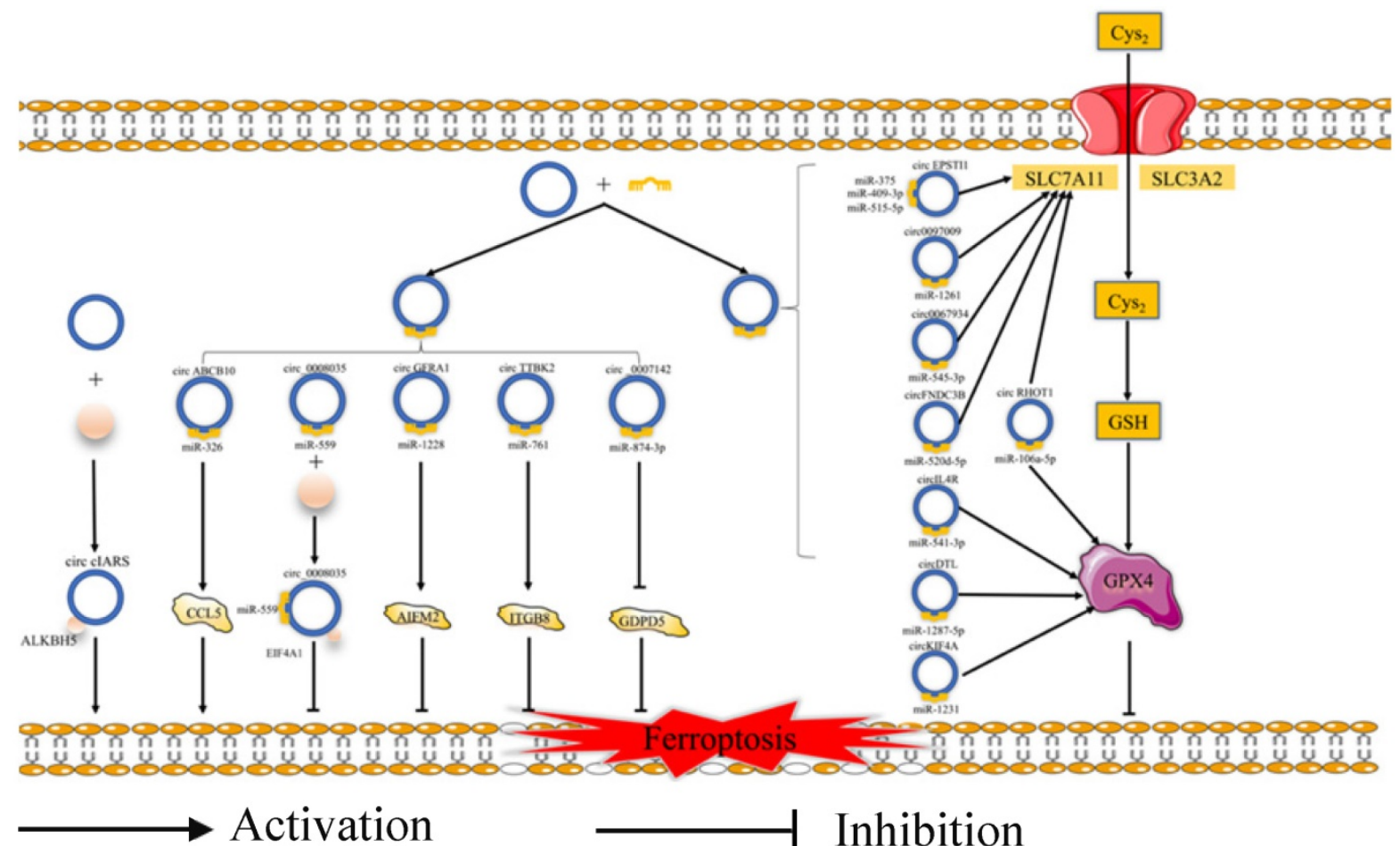

Fig. 3. The regulatory role of circRNAs in ferroptosis. Cys: Cysteine; GSH: Reduced glutathione; SLC3A2: Solute carrier family 3 membrane 2; SLC7A11: Solute carrier family 7 membrane 11; GPX4: Glutathione peroxidase 4; ALKBH5: Alk B homologue 5; CCL5: Chemokine (C-C motif) ligand 5; ElF4A1: Eukaryotic Translation Initiation Factor 4A1; ITGB8: Integrin beta 8; GDPD5: Glycerophosphodiester phosphodiesterase domain containing 5.

Table 1. The regulatory role of ncRNAs in cancer progression via ferroptosis of cancer cells.

\begin{tabular}{|c|c|c|c|c|}
\hline Cancers & NcRNAs & Mechanism & Function & Reference \\
\hline \multirow[t]{10}{*}{ Lung cancer } & exo-miR-4443 & Target METTL3/FSP1 & Induce ferroptosis and increase cisplatin resistance of NSCLC & [67] \\
\hline & miR-324-3p & Target GPX & Induce ferroptosis and reduce cisplatin resistance & [88] \\
\hline & $\operatorname{miR}-27 a-3 p$ & Target SLC7A11 & Induce ferroptosis and inhibit lung cancer & [86] \\
\hline & IncRNA P53RRA & Interact with G3BP1 & Induce ferroptosis and inhibit lung cancer & [77] \\
\hline & LINC00336 & Sponge with miR- 6825 as ceRNA & Inhibit ferroptosis and promoting lung cancer & [82] \\
\hline & lncRNA mir503HG & Sponge with miR-1273c as ceRNA & Induce ferroptosis and inhibit NCSLC & [103] \\
\hline & lncRNA NEAT1 & Upregulate the expression of ACSL4 & Induce ferroptosis and inhibit NCSLC & [96] \\
\hline & lncRNA MT1DP & target miR-365a-3p/NRF2 & Induce ferroptosis and inhibit NCSLC & [72] \\
\hline & IncRNA ASMTL-AS1 & Stabilzing SAT1 and recruiting U2AF2 & Induce ferroptosis and inhibit NCSLC & [104] \\
\hline & circDTL & Target miR-1287-5p/GPX4 & Inhibit ferroptosis and promote lung cancer & [108] \\
\hline \multirow[t]{8}{*}{ Gastrointestinal cancer } & $\operatorname{miR}-4715-3 p$ & $\begin{array}{l}\text { DNA methylation and downregulate } \\
\text { AURKA }\end{array}$ & Induce ferroptosis and inhibit UGC & [75] \\
\hline & exo-miR-522 & Interact with ALOX15 & $\begin{array}{l}\text { Inhibit ferroptosis and promote chemotherapeutic resistance of } \\
\text { GC }\end{array}$ & [80] \\
\hline & miR-103a-3p & Target GLS2 & Induce ferroptosis and inhibit GC & [94] \\
\hline & miR-139-5p & Target SLC7A11 & Induce ferroptosis and inhibit pancreatic carcinoma & [109] \\
\hline & $\operatorname{miR}-375$ & Target SLC7A11 & Induce ferroptosis and inhibit GC & [110] \\
\hline & circ_0008035 & Target miR-599/EIF4A1 & Inhibit ferroptosis and promote GC & [68] \\
\hline & $\operatorname{circABCB10}$ & Target miR-326/CCL5 & Induce ferroptosis and inhibit rectal cancer & [71] \\
\hline & circ_0007142 & Target miR-874-3p/GDPD5 & Induce ferroptosis and inhibit CRC & [70] \\
\hline \multirow[t]{6}{*}{ Liver cancer } & $\operatorname{miR}-214-3 p$ & Target ATF4 & Induce ferroptosis and inhibit $\mathrm{HCC}$ & [74] \\
\hline & lncRNA GABPB1-AS1 & Downregulation of GABPB1 and PRDX5 & Induce ferroptosis and inhibit HCC & [69] \\
\hline & lncRNA PVT1 & Target miR-214-3p/GPX4 & Inhibit ferroptosis and promote liver cancer & [90] \\
\hline & circIL4R & Target miR-541-3p/GPX4 & Inhibit ferroptosis and promote $\mathrm{HCC}$ & [91] \\
\hline & circcIARS & Target ALKBH5 & Induce ferroptosis and inhibit HCC after SF treatment & [126] \\
\hline & circ_0097009 & Target miR-1261/SLC7A11 & Inhibit ferroptosis and promote $\mathrm{HCC}$ & [84] \\
\hline \multirow[t]{5}{*}{ Breast cancer } & $\operatorname{miR}-324-3 p$ & Target GPX4 & Induce ferroptosis and inhibit breast cancer & [89] \\
\hline & miR-5096 & Target SLC7A11 & Induce ferroptosis and inhibit breast cancer & [127] \\
\hline & lncRNA P53RRA & Interact with G3BP1 & Induce ferroptosis and inhibit breast cancer & [77] \\
\hline & circGFRA1 & Target miR-1228/AIFM2 & Inhibit ferroptosis and promote breast cancer & [130] \\
\hline & circRHOT1 & Target miR-106a-5p/STAT3 & Inhibit ferroptosis and promote breast cancer & [73] \\
\hline Urogenital cancer & lncRNA RP11-89 & Target miR-129-5p/PROM2 & Inhibit ferroptosis and promote urogenital cancer & [132] \\
\hline Prostate cancer & lncRNA OIP5-AS1 & Target miR-128-3p/SLC7A11 & Inhibit ferroptosis and promote prostate cancer & [135] \\
\hline Cervical cancer & circEPSTI1 & Target miR-375/409-3P/515-5p/SLC7A11 & Inhibit ferroptosis and promote cervical cancer & [85] \\
\hline Ovarian cancer & $\operatorname{miR}-424-5 p$ & Target ACSL4 & Inhibit ferroptosis and promote ovarian cancer & [97] \\
\hline
\end{tabular}




\begin{tabular}{llll}
\hline Cancers & NcRNAs & Mechanism & Function \\
\hline $\begin{array}{l}\text { Acute myeloid } \\
\text { leukemia }\end{array}$ & LINC00618 & Downregulate SLC7A11 & Induce ferroptosis and inhibit Acute myeloid leukemia \\
Glioma & miR-18a & Downregulate ALOXE3 & Inhibit ferroptosis and promote glioma \\
& circTTBK2 & Target miR-761/ITGB8 & Inhibit ferroptosis and promote glioma \\
$\begin{array}{l}\text { Head and neck } \\
\text { squamous cell }\end{array}$ & miR-125b-5p & Target SLC7A11 & Inhibit ferroptosis and promote tongue squamous cell carcinoma [87] \\
carcinoma & circFNDC3B & Target miR-520d-5p/SLC7A11 & Inhibit ferroptosis and promote orall squamous cell carcinoma $\quad$ [78] \\
& circKIF4A & Target miR-1231/GPX4 & Inhibit ferroptosis and promote papillary thyroid cancer \\
Melanoma & circ_0067934 & Target miR-545-3p/SLC7A11 & Inhibit ferroptosis and promote thyroid cancer \\
& miR-137 & Downregulate SLC1A5 & Induce ferroptosis and inhibit melanoma \\
$\begin{array}{l}\text { Clinically relevant } \\
\text { radioresistance }\end{array}$ & miR-9 & Downregulate GOT1 & inhibit ferroptosis and promote melanoma \\
\hline
\end{tabular}

Recently, miRNAs have attracted substantial attention in lung cancer. Several miRNAs play important roles in chemotherapeutic resistance via ferroptosis. For example, exosomal miR-4443 could increase cisplatin resistance to reduce the therapeutic effect of NSCLC via METTL3/FSP1-induced ferroptosis [67]. Moreover, Shi-hua Deng [88] demonstrated that miR-324-3p could reduce cisplatin resistance by inducing GPX-mediated ferroptosis in ADC cells. Research revealed that miR-27a-3p could induce ferroptosis by suppressing the expression of SLC7A11 in NSCLC [86]. The regulatory role of miRNAs in ferroptosis might contribute to an in-depth understanding of the mechanism of chemoresistance in NSCLC and uncover potential therapeutic methods for improving chemotherapeutic resistance in NSCLC.

Furthermore, lncRNAs also play relevant roles in lung cancer progression. In 2018, Chao Mao [77] demonstrated that the lncRNA P53RRA could directly interact with the functional domain of G3BP1, resulting in abnormal accumulation of p53 in the nucleus to induce cell arrest and ferroptosis and then inhibit lung cancer progression. In contrast, it was reported that a novel IncRNA, LINC00336, which sponges miR-6825, served as a competing endogenous RNA (ceRNA) and promoted lung cancer proliferation by inhibiting ferroptosis [82]. In terms of NSCLC, some studies revealed that several ferroptosis-associated lncRNAs could inhibit tumor deterioration via ferroptosis. For instance, sponging lncRNA miR-503HG with miR-1273c may inhibit NSCLC progression via ferroptosis [103]. Similarly, Hong-xia $\mathrm{Wu}$ [96] showed that lncRNA NEAT1 upregulated the expression of ACSL4 associated with ferroptosis and inhibited the worsening of NSCLC. Another study uncovered that IncRNA MT1DP enclosed by folate-modified liposome nanoparticles via miR-365a-3p/NRF2 could improve the sensitivity to ferroptosis and might become a new therapeutic method for NSCLCs [72]. This research revealed that nanoparticles could be used to treat cancers by interacting with ncRNAs. Regarding ADC, lncRNA ASMTL-AS1 could inhibit cancer progression and promote ferroptosis by stabilizing SAT1 via recruiting
U2AF2 [104]. In addition, several ferroptosis- and iron metabolism-related lncRNAs have been identified as prognostic biomarkers of ADC $[105,106]$. Several studies have focused on the role of circRNAs in NSCLC $[107,108]$. For example, circDTL reduced the ferroptosis of cancer cells via the miR-1287-5p/GPX4 pathway [108].

However, more evidence is needed to explore the regulatory mechanism of ncRNAs in lung cancer via the ferroptosis pathway, and the regulatory roles of other ncRNAs in ferroptosis in lung cancers remain to be discovered.

\subsection{Gastrointestinal cancer}

Gastrointestinal cancer is also a serious leading cause threatening the health of humans worldwide, and its death rate is estimated to be 1.69 million $(17.1 \%)$ [99]. Its type is divided into upper gastrointestinal cancers (UGCs), including gastric cancer (GC), and lower gastrointestinal tumors, including colorectal cancer (CRC). Due to the lack of adequate knowledge of early symptoms of gastrointestinal cancer, most patients are apt to miss the optimum therapeutic period in the early stage. Thus, the disease has a huge adverse impact on family and society. Therefore, it is essential to deeply explore the pathological mechanism of gastrointestinal cancer and uncover novel diagnostic markers or therapeutic methods to diagnose or treat it in the early stage.

To explore the molecular mechanism of UGC, Ahmed Gomma [75] revealed that overexpression of miR-4715-3p could reduce Aurora kinase A levels, leading to G2/M delay of cells, and inhibiting GPX4 resulted in the ferroptosis of UGC cells. Moreover, miR-139 could inhibit SLC7A11-mediated ferroptosis via the PI3K/Akt signaling pathway and suppress the prolifetation of pancreatic carcinoma [109]. miR-375 could reduce the regeneration ability of GC cells by triggering SLC7A11-mediated ferroptosis [110]. In terms of chemotherapeutic resistance in GC, some evidence has revealed that miRNAs play an important role in chemotherapeutic resistance [80,111]. For example, exo-miR-522 secreted by cancer-associated fibroblasts interacted with ALOX15 to promote acquired chemotherapeutic resistance in GC by 
inhibiting ferroptosis in GC cells [80]. Moreover, Ying Niu [94] revealed that physcion 8-O- $\beta$ glucopyranoside, a chemical component contained in Rumex japonicas Houtt, could induce ferroptosis and suppress the proliferation and metastasis of GC cells via the miR-103a-3p/GLS2 pathway and inhibit the growth and metastasis of GC.

Not only do miRNAs play regulatory roles in the proliferation and metastasis of gastrointestinal cancer, but lncRNAs also have important effects on improving gastrointestinal cancer progression $[112,113]$. For example, Hua-jun Cai [114] investigated and constructed seven ferroptosis-related lncRNA signature by a Cox regression model to predict the survival of colon adenocarcinoma patients. An increasing number of ferroptosis-related lncRNAs may provide new insight into exploring the mechanisms of ferroptosis in GC cells and predicting GC patients [115-117]. Furthermore, circRNAs are involved in GC progression and may become novel therapeutic targets for the prevention and treatment of GC [118]. Chang Li [68] identified that circ_0008035 could promote GC cell proliferation and decrease iron accumulation and lipid peroxidation, resulting in the inhibition of cell ferroptosis via the miR-599/EIF4A1 axis in GC cells. This finding may contribute to the discovery of novel therapeutic targets for GC. Moreover, circRNAs act as regulators in the progression of CRC via ferroptosis [119]. For instance, downregulation of circABCB10 via the miR-326/ CCL5 axis promoted cancer cell ferroptosis and inhibited the progression of rectal cancer [71]. Similarly, another study [70] showed that circ_0007142 was upregulated in CRC, and inhibition of circ_0007142 could promote apoptosis and ferroptosis of CRC cells, resulting in reduced cancer cell proliferation.

\subsection{Liver cancer}

In addition to gastrointestinal cancer, liver cancer is the third largest cause of cancer-triggered death, with approximately $8.3 \%$ in both sexes [99]. The pathological types of liver cancer include hepatocellular carcinoma (HCC), cholangiocellular carcinoma, and mixed type. HCC is the most common pathological type of liver cancer. According to Barcelona Clinic Liver Cancer, the great majority of HCC patients are diagnosed after symptoms develop [120]. If they miss the therapeutic window in the early stage, the disease will be very difficult to treat. Hence, it is necessary to study the pathogenesis of liver cancer more deeply, especially regarding moderate or advanced liver cancers, and explore novel therapeutic methods or accessible diagnostic biomarkers.
Several studies have focused on ferroptosis in liver cancer and explored its molecular mechanism in depth in recent years. Some studies have revealed the role of ncRNAs in the regulation of ferroptosis in HCC [69,74]. Hence, Tao Bai [74] revealed that miR-214-3p (miR-214) could enhance erastin-induced ferroptosis by targeting ATF4 in HCC. In this research, overexpression of pre-miR-214 increased the levels of malondialdehyde (MDA), $\mathrm{ROS}$, and $\mathrm{Fe}^{2+}$, and reduced the GSH levels when HepG2 and Hep3B cells were treated with erastin, whereas overexpression of anti-miR-214 demonstrated the opposite effect. In addition, some studies began to focus on the role of lncRNAs in the regulation of cellular ferroptosis in HCC. In 2019, Wenchuan Qi et al. [69] found that lncRNA GABPB1-AS1 could reduce GABPB1 protein levels by inhibiting GABPB1 translation during erastin-induced ferroptosis in HCC cells, resulting in the downregulation of PRDX5 protein. When PRDX5 localization in mitochondria to reduce peroxidases and hydroperoxides [121] is inhibited, the completeness of the cellular membrane and cell viability are destroyed. Moreover, it was revealed that lncRNA PVT1 played an important role in accelerating the expression of GPX4 and inhibiting ferroptosis by the miR-214-3p/GPX4 pathway [90]. To identify biomarkers of prognostic prediction in HCC, many ferroptosis-related lncRNAs were validated and might become signatures for predicting the overall survival of HCC patients and novel therapeutic targets to affect HCC cell proliferation and invasion [122-125].

Similar to the roles of miRNAs and lncRNAs in liver cancer, circRNAs also demonstrate crucial regulatory roles in liver cancer cell ferroptosis. One study revealed that circIL4R, which is greatly upregulated in HCC tissues and cells, could inhibit ferroptosis by sponging miR-541-3p through the GPX4 pathway, resulting in the promotion of HCC tumorigenesis [91]. Similarly, one report from Zhiqian Liu [126] demonstrated that circcIARS was abnormally overexpressed after sorafenib treatment and could promote sorafenib-induced ferroptosis in HCC cells by inhibiting RNA-binding protein ALKBH5-induced autophagy inhibition. Moreover, circRNAs can act as ceRNAs to regulate liver cell ferroptosis. Ning Lyu [84] identified circ0097009 as a ceRNA that sponged miR-1261 and upregulated the expression of SLC7A11, inhibiting HCC cell ferroptosis and promoting the invasion and metastasis of HCC cells.

Overall, ncRNAs may become potential therapeutic targets to treat HCC via the ferroptosis pathway. 


\subsection{Breast cancer}

Breast cancer has exceeded lung cancer as the most frequently newly diagnosed cancer in both sexes and has the most cancer-related deaths among women worldwide. There were an estimated 2.3 million new cases $(11.7 \%)$ in both sexes and 0.68 million deaths $(15.5 \%)$ in females [99]. There are many therapeutic methods, including surgery, chemotherapy, and radiotherapy, to treat breast cancer. Several studies have focused on the ferroptosisrelated genes as biomarkers for diagnosing or treating and predicting prognosis in breast cancer [66]. However, reducing the number of newly diagnosed cases and mortality due to breast cancer still presents huge challenges.

To improve the therapeutic effects of drugs, several studies have revealed that some drugs exhibit anticancer effects by regulating ncRNA expression to affect cancer cell ferroptosis [89]. In this research, Yifeng Hou [89] discovered that metformin, a widely used antidiabetic drug, could lead to ferroptosis by upregulating miR-324-3p expression and downregulating GPX4 in breast cancer. This finding suggested that metformin could become a potential anticancer drug. Another study discovered that miR-5096 could increase the ROS, iron accumulation and lipid peroxidation by inhibiting SLC7A11 and inducing ferroptosis [127]. In addition to miRNAs, some lncRNAs have been identified and play an important role in regulating the ferroptosis and cancer. For instance, Chao Mao [77] found that IncRNA P53RRA not only suppressed the progression of lung cancer, but also inhibited breast cancer growth by promoting cell ferroptosis. The exploration of breast cancer biomarkers for predicting prognosis might be helpful to improve therapeutic strategies via bioinformatic analysis. Many ferroptosis-related lncRNAs have been discovered and may become prognostic signatures or potential therapeutic targets for breast cancer $[128,129]$. In addition, Huiming Zhang and his colleague [73] discovered that circRHOT1 could attenuate cancer cell ferroptosis through the miR-106a-5p/STAT3 pathway and promote the invasion and migration of breast cancer cells, leading to worsening progression of breast cancer. In HER-2-positive breast cancer, circGFRA1 could inhibit ferroptosis and promote cancer progression via the miR-1228/AIFM2 axis [130]. These findings may provide new insight into exploring the regulatory mechanism. However, more studies are still needed to focus on the regulatory role of ncRNAs in breast cancer via ferroptosis.

\subsection{Urogenital cancer}

Urogenital cancer is also one of most common cancers, but its newly diagnosed cases and deaths are lower than those of female breast cancer. It consists of bladder cancer, renal cancer, ureteropelvic cancer and urinary tract cancer. Among these cancers, bladder cancer and renal cancer accounted for an estimated 1 million new cases (5.5\%) and 0.37 million deaths $(3.9 \%)$ worldwide [99]. Although there are some therapeutic methods to treat it, exploring the novel diagnostic and therapeutic methods is still essential.

To explore novel diagnostic and therapeutic targets, accumulating evidence has focused on the role of ferroptosis-related ncRNAs in the genesis, progression, and treatment of urogenital cancer [131]. In term of bladder cancer, lncRNA RP11-89 could induce tumorigenesis and reduce the accumulation of cellular iron by sponging the miR-129-5p/PROM2 pathway, and leading to ferroptosis inhibition [132]. Moreover, several studies have revealed that some ferroptosis-associated lncRNAs could become prognostic signatures in renal clear cell carcinoma [133]. More studies are needed to uncover more ferroptosis-related ncRNAs and explore their regulatory role of them in the urogenital cancer.

\subsection{Prostate cancer}

The number of new cases of prostate cancer is very high, next only to lung cancer in males, at an estimated $14.1 \%$, but the cancer-related death rate is lower (approximately 4.5\%) than that of lung cancer (approximately 21.5\%) [99]. The occurrence and progression of prostate cancer involve both genetic and environmental factors [134]. In a recent study, Yangyi Zhang [135] demonstrated that chronic cadmium exposure could promote cancer cell growth and inhibit ferroptosis by upregulating lncRNA OIP5-AS1 expression, and lncRNA OIP5-AS1 acted as a ceRNA that sponges miR-128-3p to increase the level of SLC7A11. However, future studies are needed to reveal the regulatory role of ferroptosis-related ncRNAs in inhibiting the progression of prostate cancer.

\subsection{Cervical cancer}

Cervical cancer is the fourth most common cancer in women, with an estimated $6.5 \%$, and ranks as the fourth leading cause of cancer-associated death in women, with approximately $7.7 \%$ [85,99]. Although there are many therapeutic methods to treat it, such as chemotherapy and surgery, cervical cancer still lacks adequate and effective treatment to improve the low survival rate and poor prognosis. Recent studies have focused on the potential regulatory role of ncRNAs in improving the prognosis of cervical cancer via ferroptosis. For example, Peng $\mathrm{Wu}$ [85] showed that circEPSTI1 promoted the proliferation of cervical 
cancer via miR-375/409-3P/515-5p acting as a ceRNA by targeting SLC7A11 and attenuated the effect of lipid peroxidation and GSH/GSSG to inhibit ferroptosis of cervical cancer cells.

\subsection{Ovarian cancer}

Ovarian cancer is also a common gynecological cancer, with its number of deaths ranking eighth among gynecological cancers [99]. The majority of ovarian cancers cannot be diagnosed at an early stage, and the 5-year survival rate is low. More studies should be conducted to discover the pathogenic mechanism of ovarian cancer. Currently, some evidence has revealed that ncRNAs play an important role in suppressing ovarian cancer via the ferroptosis pathway $[136,137]$. Research revealed that miR-424-5p negatively regulated ferroptosis by directly targeting ACSL4, an overexpressed ferroptosis-related protein, in ovarian cancer cells and that downregulation of miR-424-5p increased erastin- and RSL3-induced ferroptosis, resulting in inhibition of the progression of ovarian cancer [97].

\subsection{Acute myeloid leukemia}

Acute leukemia (AL), including acute myeloid leukemia (AML) and acute lymphoblastic leukemia (ALL), is also a serious malignant disease [138]. AL is a type of malignant clonal disease stemming from hematopoietic stem cells, and AML is very common in adults [138]. The five-year survival rates of patients treated with chemotherapeutics were $27.4 \%$ [139] lower than those of patients treated with stem cell transplantations. Moreover, drug resistance is one of the major problems in chemotherapy for AML. Therefore, it is necessary to explore the mechanism of drug resistance to increase the therapeutic sensitivity of chemotherapy in AML. The abnormal expression of ncRNAs may be the key regulator to improve drug resistance [140]. For example, Zuili Wang and his colleague [79] revealed that lncRNA LINC00618 was downregulated in human leukemia and strongly increased by VCR therapeutics, and it was involved in inducing AML cell ferroptosis by increasing the production of ROS and iron and decreasing the expression of SLC7A11 in AML.

However, ncRNAs, including miRNAs, lncRNAs and circRNAs, require further investigation regarding the chemotherapeutic resistance of AML.

\subsection{Glioma}

Although less common in other cancers, glioma is the most common primary central nervous system tumor and accounts for approximately half of all primary intracranial tumors. The five-year survival rate of adult high-grade glioma is very low. According to the WHO grade, low-grade glioma is grade I or II, and high-grade glioma is grade III or IV among the four grades of glioma [141]. Grade IV glioma is also named glioblastoma (GBM). Hence, it is essential to explore the regulatory mechanism of glioma and some novel therapeutic methods. Recently, some studies have reported that some ncRNAs play important roles in inhibiting glioma via ferroptosis [76,98]. Xinzhi Yang [98] revealed that miR-18a accelerated glioblastoma advancement by directly inhibiting ALOXE3-mediated ferroptotic and antimigration activities. Deficiency of ALOXE3 in GBM cells results in resistance to p53-SLC7A11associated ferroptosis and improves the survival rate of GBM cells. Moreover, it was reported that the circRNA TTBK2 was upregulated in glioma tissues and cells and inhibited ferroptosis via the miR-761/ITGB8 axis to promote glioma proliferation and invasion [76].

\subsection{Head and neck squamous cell carcinoma}

Head and neck squamous cell carcinoma (HNSCC) is a series of malignant tumors involving many tissues in the head and neck region, including the oral cavity, nasopharynx and throat [142]. Although surgery, radiotherapy and chemotherapy are available, the morbidity of HNSCC has increased markedly in recent years, especially in women [99]. It is particularly important to explore the regulatory mechanism and uncover novel remarkable biomarkers and therapeutic targets to improve the progression of HNSCC. Bin Zhang revealed that miR-125b-5p could inhibit the expression of SLC7A11 and that enhancer of zeste homolog 2 (EZH2) inhibited ferroptosis via the miR-125b-5p/SLC7A11 pathway in tongue squamous cell carcinoma [87]. Moreover, Yun Tang [143] identified that some ferroptosis-related lncRNAs may become diagnostic biomarkers and potential therapeutic targets to treat HNSCC via ferroptosis. Furthermore, some research has focused on the role of ferroptosis-related circRNAs in HNSCC. For example, circFNDC3B could inhibit cancer cell ferroptosis by miR-520d-5p/ SLC7A11 pathway in oral squamous cell carcinoma [78]. Another study revealed that circKIF4A could upregulate the levels of GPX4 and reduce the ferroptosis of thyroid cancer cells by sponging miR-1231, and leading to the induction of cancer progression [92]. Moreover, circ_0067934 reduced lipid peroxidation and ferroptosis of thyroid cancer cells via the miR-545-3p/SLC7A11 pathway [144]. However, more evidence is needed to identify the regulatory roles of these ncRNAs in HNSCC via ferroptosis. 


\subsection{Melanoma}

In contrast to other cancers, melanoma stemming from melanocytes is not common. However, melanoma is the third most frequently occurring malignant tumor of the skin. Melanoma lacks specific treatment, except for surgical resection in the early stage. Hence, ferroptosis is a focus of researchers, and new cell death contributes to inhibiting melanoma progression. Meiying Luo [81] revealed that miR-137 could inhibit glutamine transporter SLC1A5, an inhibitor of ferroptosis, in melanoma cells and that the suppression of SLC1A5 decreased glutamine uptake and MDA accumulation, resulting in ferroptosis and inhibiting the progression of melanoma. In another study, Kexin Zhang [95] identified that overexpression of miR-9 inhibited GOT1, leading to reduced erastin- and RSL3-mediated ferroptosis. Suppression of miR-9 could increase the levels of lipid ROS in melanoma cells, leading to promotion of ferroptosis in melanoma cells and the inhibition of melanoma growth [95]. However, other ncRNAs, including lncRNAs and circRNAs, ought to be focused on to define the regulatory role of ferroptosis in melanoma.

\subsection{Clinically relevant radioresistance}

Like chemotherapy, radiation therapy (RT) is also one of the most common therapies for cancers. However, radioresistance decreases the therapeutic effect of RT, and the mechanism of radioresistance is not well understood. In recent years, Kazuo Tomita [83] revealed that miR-7-5p played a crucial role in regulating irradiation resistance by controlling intracellular $\mathrm{Fe}^{2+}$ content in clinically relevant radioresistant (CRR) cells. Oxidative stress and ferroptosis in CRR cells are inhibited. In the future, more investigations will be required to uncover the mechanism by which miRNAs improve radioresistance in cancer cells via the ferroptosis pathway.

\section{Conclusions and future prospects}

Ferroptosis, a newly discovered form of programmed cell death, is related to some pathophysiological processes, especially many types of cancers. Numerous studies have focused on exploring the regulatory mechanisms of cancers via the ferroptosis pathway. Great progress in exploring the regulatory role of ncRNAs in cancers by ferroptosis has been made. Taken together, these findings contribute to further understanding the pathogenesis of cancers and have demonstrated that ferroptosis-associated ncRNAs may act as a series of tumor inhibitors to suppress cancer growth. In addition, ncRNAs, including miRNAs, lncRNAs and
circRNAs, have the potential to be novel anticancer therapeutic methods and diagnostic biomarkers by regulating the ferroptosis of cancer cells (Table 2).

Table 2. The therapeutic target and diagnostic or prognostic biomarker of cancers.

\begin{tabular}{|c|c|c|}
\hline Cancers & Therapeutic Target & $\begin{array}{l}\text { Diagnostic or Prognostic } \\
\text { Biomarker }\end{array}$ \\
\hline \multirow[t]{14}{*}{ Lung cancer } & exo-miR-4443 [67] & miR-27a-3p [86] \\
\hline & miR-324-3p [88] & IncRNA C20orf197 [105] \\
\hline & miR-27a-3p [86] & IncRNA ARHGEF26-AS1 [105] \\
\hline & LINC00336 [82] & IncRNA MGC32805 [105] \\
\hline & IncRNA NEAT1 [96] & IncRNA LINC00324 [105] \\
\hline & lncRNA MT1DP [72] & IncRNA LINC01116 [105] \\
\hline & circDTL [108] & IncRNA LINC01137 [105] \\
\hline & - & IncRNA TMPO-AS1 [105] \\
\hline & - & IncRNA AC021016.1 [106] \\
\hline & - & IncRNA AC068228.2 [106] \\
\hline & - & IncRNA MIR223HG [106] \\
\hline & - & IncRNA AC009275.1 [106] \\
\hline & - & IncRNA AL049555.1 [106] \\
\hline & - & IncRNA KTN1-AS1 [106] \\
\hline \multirow{9}{*}{$\begin{array}{l}\text { Gastrointestinal } \\
\text { cancer }\end{array}$} & exo-miR-522 [80] & $\operatorname{miR}-375[110]$ \\
\hline & miR-103a-3p [94] & LINC01503 [114] \\
\hline & miR-139-5p [109] & IncRNA AC004687.1 [114] \\
\hline & circ_0008035 [68] & IncRNA AC010973.2 [114] \\
\hline & circABCB10 [71] & IncRNA AP001189.3 [114] \\
\hline & circ_0007142 [70] & IncRNA ARRDC1-AS1 [114] \\
\hline & - & IncRNA OIP5-AS1 [114] \\
\hline & - & IncRNA NCK1-DT [114] \\
\hline & - & - \\
\hline \multirow[t]{26}{*}{ Liver cancer } & miR-214-3p [74] & IncRNA RHPN1-AS1 [122] \\
\hline & IncRNA GABPB1-AS1 [69] & $\begin{array}{l}\text { IncRNA MAPKAPK5-AS1 } \\
{[122]}\end{array}$ \\
\hline & circIL4R [91] & lncRNA PART1 [122] \\
\hline & circ_0097009 [84] & LINC00324 [123] \\
\hline & - & lncRNA MSC-AS1 [123] \\
\hline & - & lncRNA AC023157.3 [123] \\
\hline & - & IncRNA AC0090005.1 [123] \\
\hline & - & IncRNA PRRT3-AS1 [123] \\
\hline & - & IncRNA AC015908.3 [123] \\
\hline & - & IncRNA AC145207.5 [123] \\
\hline & - & IncRNA AL031985.3 [123] \\
\hline & - & IncRNA TMEM220-AS1 [123] \\
\hline & - & IncRNA LUCAT1 [124] \\
\hline & - & IncRNA AC099850.3 [124] \\
\hline & - & IncRNA AL365203.2 [124] \\
\hline & - & IncRNA AL031985.3 [124] \\
\hline & - & IncRNA CTD-2033A16.3 [125] \\
\hline & - & IncRNA CTD-2116N20.1 [125] \\
\hline & - & IncRNA CTD-2510F5.4 [125] \\
\hline & - & IncRNA DDX11-AS1 [125] \\
\hline & - & IncRNA ZFPM2-AS1 [125] \\
\hline & - & LINC00942 [125] \\
\hline & - & LINC01224 [125] \\
\hline & - & LINC01231 [125] \\
\hline & - & LINC01508 [125] \\
\hline & - & circ_0097009 [84] \\
\hline \multirow[t]{13}{*}{ Breast cancer } & miR-5096 [127] & IncRNA AC10874.1 [128] \\
\hline & circRHOT1 [73] & IncRNA AL133467.1 [128] \\
\hline & circGFRA1 [130] & LINC01235 [128] \\
\hline & - & IncRNA AC072039.2 [128] \\
\hline & - & lncRNA TDRKH-AS1 [128] \\
\hline & - & lncRNA USP30-AS1 [128] \\
\hline & - & IncRNA MAPT-AS1 [128] \\
\hline & - & IncRNA LIPE-AS [128] \\
\hline & - & IncRNA AC121247.2 [129] \\
\hline & - & IncRNA LIPE-AS1 [129] \\
\hline & - & IncRNA HSD11B1-AS1 [129] \\
\hline & - & IncRNA AC010655.2 [129] \\
\hline & - & IncRNA PTPRD-AS1 [129] \\
\hline
\end{tabular}




\begin{tabular}{|c|c|c|}
\hline Cancers & Therapeutic Target & $\begin{array}{l}\text { Diagnostic or Prognostic } \\
\text { Biomarker }\end{array}$ \\
\hline \multirow{10}{*}{ Urogenital cancer } & - & lncRNA AC099329.2 [129] \\
\hline & - & lncRNA OTUD6B [129] \\
\hline & - & LINC01871 [129] \\
\hline & - & LINC00393 [129] \\
\hline & - & LINC02384 [129] \\
\hline & - & LINC01419 [129] \\
\hline & - & LINC02266 [129] \\
\hline & lncRNA RP11-89 [132] & lncRNA DUXAP8 [133] \\
\hline & - & lncRNA LUCAT1 [133] \\
\hline & - & LINC02609 [133] \\
\hline Prostate cancer & IncRNA OIP5-AS1 [135] & - \\
\hline Cervical cancer & circEPSTI1 [85] & circEPSTI1 [85] \\
\hline Ovarian cancer & miR-424-5p [97] & - \\
\hline \multirow[t]{2}{*}{ Glioma } & miR-18a [98] & miR-18a [98] \\
\hline & circTTBK2 [76] & - \\
\hline \multirow{9}{*}{$\begin{array}{l}\text { Head and neck } \\
\text { squamous cell } \\
\text { carcinoma }\end{array}$} & miR-520d-5p [78] & LINC01963 [143] \\
\hline & miR-125b-5p [87] & \\
\hline & LINC01963 [143] & LINC01980 [143] \\
\hline & LINC01980 [143] & IncRNA AATBC [143] \\
\hline & lncRNA AATBC [143] & IncRNA ELF3-AS1 [143] \\
\hline & lncRNA ELF3-AS [143] & - \\
\hline & circFNDC3B [78] & - \\
\hline & circKIF4A [92] & - \\
\hline & circ_0067934 [144] & - \\
\hline \multirow[t]{2}{*}{ Melanoma } & $\operatorname{miR}-137$ [81] & - \\
\hline & $\operatorname{miR}-9$ [95] & - \\
\hline $\begin{array}{l}\text { Clinically relevant } \\
\text { radioresistance }\end{array}$ & miR-7-5p [83] & - \\
\hline
\end{tabular}

Many studies have revealed that these ncRNAs play important roles in the progression of cancers via ferroptosis and that these ncRNAs may regulate the ferroptosis of cancer cells to induce or inhibit tumorigenesis. It has been demonstrated that lncRNAs and circRNAs always sponge miRNAs to regulate the expression of GPX4 and induce lipid peroxidation of the cellular membrane. Furthermore, abnormal lipid peroxidation destroys cancer cell membranes, resulting in the ferroptosis of cancer cells. Sometimes, these ncRNAs can directly target GPX4, SLC3A2 and SLC7A11 to induce ferroptosis in cancer cells. In addition, some ncRNAs regulate the ferroptosis of cancer cells via the lethal accumulation of ROS and the abnormal metabolism of iron.

Due to the individual heterogeneity of ncRNA expression in different types of cancer, ncRNAassociated therapy and biomarkers may be applied to support personalized cancer treatment. Although an increasing number of studies have revealed a regulatory mechanism between cancer and ferroptosis, a deeper understanding of the mechanism by which ferroptosis-related ncRNAs regulate the progression and growth of cancer is still needed. Moreover, future studies should pay more attention to the role of ncRNAs in the linkage between cancer and ferroptosis. Moreover, some biomaterials, like nanomaterials, may overcome the shortcomings of conventional therapeutic schedules for tumortargeted ferroptosis therapy by preloading antitumor drugs $[145,146]$. However, the regulatory relationship between these promising materials and ferroptosis- related ncRNAs still lacks of research and should be given more attention.

This review has summarized the regulatory roles of several types of ncRNAs in cancer progression and ferroptosis. These studies are beneficial for understanding the pathogenesis of cancer. Ferroptosis-related ncRNAs have great potential to act as anticancer therapeutic targets by regulating ferroptosis. Targeting these key ncRNAs may reveal novel therapeutic methods or diagnostic biomarkers to inhibit the growth and progression of malignant tumors.

\section{Abbreviations}

ncRNAs: noncoding RNAs; PCD: programmed cell death; TFRC: transferrin receptor; SLC40A1: solute carrier family 40 membrane 1; NUPR1: nuclear protein 1; GPX4: glutathione peroxidase 4; Glu: glutamate; Cys2: cysteine; SLC3A2: solute carrier family 3 membrane 2; SLC7A11: solute carrier family 7 membrane 11; BAP1: BRCA1-related protein; KEAP1: Kelch-like ECH-associtaed protein 1; NRF2: NF E2-related factor 2; PGRMC1: progesterone receptor membrane component 1; mTORC1: rapamycin complex 1; AA: arachidonic acid; ACSL4: acyl-CoA synthetase long-chain family member 4; LPCAT3: lysophophatidylcholne acyltransferase 3; LOXs: lipoxygenases; miRNAs: microRNAs; lncRNAs: long noncoding RNAs; circRNAs: circular RNAs; GLS2: glutaminase 2; GOT1: glutamic-oxaloacetic transaminase 1; SLC1A5: solute carrier family 1 membrane 5; SCLC: small cell lung cancer; NSCLC: non-small cell lung cancer; ADC: adenocarcinoma; SCC: squamous cell carcinoma; ceRNA: competing endogenous RNA; UGCs: upper gastric cancers; CRC: colorectal cancer; HCC: hepatocellular carcinoma; MDA: malondialdehyde; AL: Acute leukemia; AML: acute myeloid leukemia; ALL: acute lymphoblastic leukemia; GBM: glioblastoma; HNSCC: head and neck squamous cell carcinoma; EZH2: enhancer of zeste homolog 2; RT: radiation therapy; CRR: clinically relevant radioresistant.

\section{Acknowledgements}

This work was supported by the People's Livelihood Science and Technology Project of Qingdao (19-6-1-7-nsh), the National Natural Science Foundation of China (22006084), the Qingdao Applied Basic Research Project (19-6-2-49-cg), and Hubei Key Laboratory of Environmental and Health Effects of Persistent Toxic Substances (PTS2019-05).

\section{Author Contributions}

Y.Z.: Wrote original draft, drew the figures and 
investigated the articles. Y.Z.: Supervision, reviewed and edited draft. M.C., Z.Z. and J.S.: drew the figures and tables. R.Z., J.T., X.L., R.L. and S.L.: investigated and analysed the articles. H.X.: Supervision and coordination.

\section{Competing Interests}

The authors have declared that no competing interest exists.

\section{References}

1. Fuchs $Y$, Steller H. Programmed cell death in animal development and disease. Cell. 2011; 147: 742-58.

2. Galluzzi L, Vitale I, Abrams JM, Alnemri ES, Baehrecke EH, Blagosklonny M V., et al. Molecular definitions of cell death subroutines: Recommendations of the Nomenclature Committee on Cell Death 2012. Cell Death Differ. 2012; 19: 107-20.

3. Jiang N, Zhang X, Gu X, Li X, Shang L. Progress in understanding the role of IncRNA in programmed cell death. Cell Death Discov. 2021; 7: 30.

4. Dixon SJ, Lemberg KM, Lamprecht MR, Skouta R, Eleina M, Gleason CE, et al. Ferroptosis: an iron-dependent form of nonapoptotic cell death. Cell. 2012; 149: 1060-72.

5. Yang WS, Stockwell BR. Ferroptosis: death by lipid peroxidation. Trends Cell Biol. 2016; 26: 165-76.

6. Chen X, Yu C, Kang R, Kroemer G, Tang D. Cellular degradation systems in ferroptosis. Cell Death Differ. 2021; 28: 1135-48.

7. Liu J, Kuang F, Kroemer G, Klionsky DJ, Kang R, Tang D. Autophagy-Dependent Ferroptosis: Machinery and Regulation. Cell Chem Biol. 2021; 27: 420-35.

8. Bao WD, Pang P, Zhou XT, Hu F, Xiong W, Chen K, et al. Loss of ferroportin induces memory impairment by promoting ferroptosis in Alzheimer's disease. Cell Death Differ. 2020; 28: 1548-62.

9. Fang X, Cai Z, Wang H, Han D, Cheng Q, Zhang P, et al. Loss of Cardiac Ferritin H Facilitates Cardiomyopathy via Slc7a11-Mediated Ferroptosis. Circ Res. 2020; 127: 486-501.

10. Chen X, Kang R, Kroemer G, Tang D. Broadening horizons: the role of ferroptosis in cancer. Nat Rev Clin Oncol. 2021; 18: 280-96.

11. Galluzzi L, Vitale I, Aaronson SA, Abrams JM, Adam D, Agostinis P, et al. Molecular mechanisms of cell death: Recommendations of the Nomenclature Committee on Cell Death 2018. Cell Death Differ. 2018; 25: 486-541.

12. Kuang F, Liu J, Tang D, Kang R. Oxidative Damage and Antioxidant Defense in Ferroptosis. Front Cell Dev Biol. 2020; 8: 586578.

13. Chen X, Yu C, Kang R, Tang D. Iron Metabolism in Ferroptosis. Front Cell Dev Biol. 2020; 8: 590226.

14. Yang WS, Stockwell BR. Synthetic Lethal Screening Identifies Compounds Activating Iron-Dependent, Nonapoptotic Cell Death in Oncogenic-RAS-Harboring Cancer Cells. Chem Biol. 2008; 15: 234-45.

15. Wang Y, Liu Y, Liu J, Kang R, Tang D. NEDD4L-mediated LTF protein degradation limits ferroptosis. Biochem Bioph Res Co. 2020; 531: 581-7.

16. Floros KV., Cai J, Jacob S, Kurupi R, Fairchild CK, Shende M, et al. MYCN -amplified neuroblastoma is addicted to iron and vulnerable to inhibition of the system xc-/glutathione axis. Cancer Res. 2021; 81: 1896-908.

17. Gao M, Monian P, Pan Q, Zhang W, Xiang J, Jiang X. Ferroptosis is an autophagic cell death process. Cell Res. 2016; 26: 1021-32.

18. Geng N, Shi BJ, Li SL, Zhong ZY, Li YC, Xua WL, et al. Knockdown of ferroportin accelerates erastin-induced ferroptosis in neuroblastoma cells. Eur Rev Med Pharmacol Sci. 2021; 22: 3826-36.

19. Hou W, Xie Y, Song X, Sun X, Lotze MT, Zeh HJ, et al. Autophagy promotes ferroptosis by degradation of ferritin. Autophagy. 2016; 12: 1425-8.

20. Nishizawa H, Matsumoto M, Shindo T, Saigusa D, Kato H, Suzuki K, et al. Ferroptosis is controlled by the coordinated transcriptional regulation of glutathione and labile iron metabolism by the transcription factor BACH1. J Biol Chem. 2020; 295: 69-82.

21. Alvarez SW, Sviderskiy VO, Terzi EM, Papagiannakopoulos T, Moreira AL, Adams S, et al. NFS1 undergoes positive selection in lung tumours and protects cells from ferroptosis. Nature. 2017; 551: 639-643.

22. Du J, Wang T, Li Y, Zhou Y, Wang X, Yu X, et al. DHA inhibits proliferation and induces ferroptosis of leukemia cells through autophagy dependent degradation of ferritin. Free Radical Bio Med. 2019; 131: 356-69.

23. Yuan H, Li X, Zhang X, Kang R, Tang D. CISD1 inhibits ferroptosis by protection against mitochondrial lipid peroxidation. Biochem Bioph Res Co. 2016; 478: 838-44.

24. Kim EH, Shin D, Lee J, Jung AR, Roh JL. CISD2 inhibition overcomes resistance to sulfasalazine-induced ferroptotic cell death in head and neck cancer. Cancer Lett. 2018; 432: 180-90.

25. Liu J, Song X, Kuang F, Zhang Q, Xie Y, Kang R, et al. NUPR1 is a critical repressor of ferroptosis. Nat Commun. 2021; 12: 647.
26. Imoto $\mathrm{S}$, Kono $\mathrm{M}$, Suzuki $\mathrm{T}$, Shibuya $\mathrm{Y}$, Sawamura $\mathrm{T}$, Mizokoshi $\mathrm{Y}$, et al. Haemin-induced cell death in human monocytic cells is consistent with ferroptosis. Transfus Apher Sci. 2018; 57: 524-31

27. Do Van B, Gouel F, Jonneaux A, Timmerman K, Gelé P, Pétrault M, et al. Ferroptosis, a newly characterized form of cell death in Parkinson's disease that is regulated by PKC. Neurobiol Dis. 2016; 94: 169-78.

28. Park E, Chung SW. ROS-mediated autophagy increases intracellular iron levels and ferroptosis by ferritin and transferrin receptor regulation. Cell Death and Dis. 2019; 10: 822.

29. Liu J, Yang M, Kang R, Klionsky DJ, Tang D. Autophagic degradation of the circadian clock regulator promotes ferroptosis. Autophagy. 2019; 15: 2033-5.

30. Yang M, Chen P, Liu J, Zhu S, Kroemer G, Klionsky DJ, et al. Clockophagy is a novel selective autophagy process favoring ferroptosis. Sci Adv. 2019; 5: eaaw2238.

31. Ingold I, Berndt C, Schmitt S, Doll S, Poschmann G, Buday K, et al. Selenium Utilization by GPX4 Is Required to Prevent Hydroperoxide-Induced Ferroptosis. Cell. 2018; 172: 409-422.

32. Ursini F, Maiorino M. Lipid peroxidation and ferroptosis: The role of GSH and GPx4. Free Radic Biol Med. 2020; 152: 175-85.

33. Brigelius-Flohé R, Maiorino M. Glutathione peroxidases. Biochim Biophys Acta. 2013; 1830: 3289-303

34. Stockwell BR, Friedmann Angeli JP, Bayir H, Bush AI, Conrad M, Dixon SJ, et al. Ferroptosis: A Regulated Cell Death Nexus Linking Metabolism, Redox Biology, and Disease. Cell. 2017; 171: 273-85.

35. Shaw AT, Winslow MM, Magendantz M, Ouyang C, Dowdle J, Subramanian A, et al. Selective killing of K-ras mutant cancer cells by small molecule inducers of oxidative stress. P Natl Acad Sci USA. 2011; 108: 8773-8.

36. Zhang Y, Shi J, Liu X, Feng L, Gong Z, Koppula P, et al. BAP1 links metabolic regulation of ferroptosis to tumor suppression. Nat Cell Biol. 2018; 20: 118192.

37. Habib E, Linher-Melville K, Lin HX, Singh G. Expression of $\mathrm{xCT}$ and activity of system xc- are regulated by NRF2 in human breast cancer cells in response to oxidative stress. Redox Biol. 2015; 5: 33-42.

38. Fan Z, Wirth AK, Chen D, Wruck CI, Rauh M, Buchfelder M, et al. Nrf2-keap1 pathway promotes cell proliferation and diminishes ferroptosis. Oncogenesis. 2017; 6: e371-14

39. Zhang W, Sun Y, Bai L, Zhi L, Yang Y, Zhao Q, et al. RBMS1 regulates lung cancer ferroptosis through translational control of SLC7A11. J Clin Invest. 2021; 131: e152067.

40. You JH, Lee J, Roh J-L. PGRMC1-dependent lipophagy promotes ferroptosis in paclitaxel-tolerant persister cancer cells. J Exp Clin Cancer Res. 2021; 40: 350.

41. Xie Y, Hou W, Song X, Yu Y, Huang J, Sun X, et al. Ferroptosis: Process and function. Cell Death Differ. 2016; 23: 369-79.

42. Ma L, Zhang X, Yu K, Xu X, Chen T, Shi Y, et al. Targeting SLC3A2 subunit of system $\mathrm{XC}-$ is essential for m6A reader YTHDC2 to be an endogenous ferroptosis inducer in lung adenocarcinoma. Free Radic Biol Med. 2021; 168: 25-43.

43. Yang WS, Sriramaratnam R, Welsch ME, Shimada K, Skouta R, Viswanathan VS, et al. Regulation of Ferroptotic Cancer Cell Death by GPX4. Cell. 2014; 156: 317-31.

44. Conrad M, Pratt DA. The chemical basis of ferroptosis. Nat Chem Biol. 2019; 15: 1137-47.

45. Zhang Y, Swanda R V., Nie L, Liu X, Wang C, Lee H, et al. mTORC1 couples cyst(e)ine availability with GPX4 protein synthesis and ferroptosis regulation. Nat Commun. 2021; 12: 1589

46. Lei G, Zhuang L, Gan B. mTORC1 and ferroptosis: Regulatory mechanisms and therapeutic potential. Bioessays. 2021; 43: e2100093.

47. Yan Y, Lei G, Gan B. A mTORC1-mediated cyst(e)ine sensing mechanism governing GPX4 synthesis and ferroptosis. Mol Cell Oncol. 2021:8: 1919006.

48. Sun Y, Berleth N, Wu W, Schlütermann D, Deitersen J, Stuhldreier F, et al. Fin56-induced ferroptosis is supported by autophagy-mediated GPX4 degradation and functions synergistically with mTOR inhibition to kill bladder cancer cells. Cell Death Dis. 2021; 12: 1028.

49. Lu Y, Qin H, Jiang B, Lu W, Hao J, Cao W, et al. KLF2 inhibits cancer cell migration and invasion by regulating ferroptosis through GPX4 in clear cell renal cell carcinoma. Cancer Lett. 2021; 522: 1-13

50. Wu Z, Geng Y, Lu X, Shi Y, Wu G, Zhang M, et al. Chaperone-mediated autophagy is involved in the execution of ferroptosis. P Natl Acad Sci USA. 2019: 116: 2996-3005.

51. Friedmann Angeli JP, Schneider M, Proneth B, Tyurina YY, Tyurin VA, et al. Inactivation of the ferroptosis regulator Gpx4 triggers acute renal failure in mice. Nat Cell Biol. 2014; 16: 1180-91.

52. Dixon SJ, Winter GE, Musavi LS, Lee ED, Snijder B, Rebsamen M, et al. Human Haploid Cell Genetics Reveals Roles for Lipid Metabolism Genes in Nonapoptotic Cell Death. ACS Chem Biol. 2015; 10: 1604-9.

53. Kagan VE, Mao G, Qu F, Friedmann Angeli JP, Doll S, et al. Oxidized Arachidonic/Adrenic Phosphatidylethanolamines Navigate Cells to Ferroptosis. Nat Chem Biol. 2017; 13: 81-90.

54. De Carvalho CCCR, Caramujo MJ. The various roles of fatty acids. Molecules. 2018; 23: 2583.

55. Yagoda N, Rechenberg M Von, Zaganjor E, Bauer AJ, Yang S, Fridman DJ, et al. RAS-RAF-MEK-dependent oxidative cell death involving voltage-dependent anion channels. Nature. 2007; 447: 864-8. 
56. Bedoui S, Herold MJ, Strasser A. Emerging connectivity of programmed cell death pathways and its physiological implications. Nat Rev Mol Cell Bio. 2020; 21: 678-95.

57. Joshi SS, Badgwell BD. Current treatment and recent progress in gastric cancer. CA Cancer J Clin. 2021; 71: 264-79.

58. Shen Z, Song J, Yung BC, Zhou Z, Wu A, Chen X. Emerging Strategies of Cancer Therapy Based on Ferroptosis. Adv Mater. 2018; 30: e1704007.

59. Roh JL, Kim EH, Jang HJ, Park JY, Shin D. Induction of ferroptotic cell death for overcoming cisplatin resistance of head and neck cancer. Cancer Lett. 2016; 381: 96-103.

60. Chen L, Li X, Liu L, Yu B, Xue Y, Liu Y. Erastin sensitizes Glioblastoma cells to temozolomide by restraining $x \mathrm{CT}$ and cystathionine- $\gamma$-lyase function. Oncol Rep. 2015; 33: 1465-74.

61. Mao C, Liu X, Zhang Y, Lei G, Yan Y, Lee H, et al. DHODH-mediated ferroptosis defence is a targetable vulnerability in cancer. Nature. 2021; 593: 586-90.

62. Lei G, Zhang Y, Koppula P, Liu X, Zhang J, Lin SH, et al. The role of ferroptosis in ionizing radiation-induced cell death and tumor suppression. Cell Res. 2020; 30: 146-62

63. Zhang X, Wang L, Li H, Zhang L, Zheng X, Cheng W. Crosstalk between noncoding RNAs and ferroptosis: new dawn for overcoming cancer progression. Cell Death Dis. 2020; 11: 580.

64. Luo Y, Huang Q, He B, Liu Y, Huang S, Xiao J. Regulation of ferroptosis by non-coding RNAs in the development and treatment of cancer. Oncol Rep. 2020; 45: 29-48.

65. Wang Y, Wei Z, Pan K, Li J, Chen Q. The function and mechanism of ferroptosis in cancer. Apoptosis. 2020; 25: 786-98

66. Zhang Z, Qiu X, Yan Y, Liang Q, Cai Y, Peng B, et al. Evaluation of ferroptosis-related gene AKR1C1 as a novel biomarker associated with the immune microenvironment and prognosis in breast cancer. Int J Gen Med. 2021; 14: 6189-200.

67. Song Z, Jia G, Ma P, Cang S. Exosomal miR-4443 promotes cisplatin resistance in non-small cell lung carcinoma by regulating FSP1 m6A modification-mediated ferroptosis. Life Sci. 2021; $276: 119399$.

68. Li C, Tian Y, Liang Y, Li Q. Circ_0008035 contributes to cell proliferation and inhibits apoptosis and ferroptosis in gastric cancer via miR-599/EIF4A1 axis. Cancer Cell Int. 2020;20: 84.

69. Oi W, Li Z, Xia L, Dai J, Zhang Q, Wu C, et al. LncRNA GABPB1-AS1 and GABPB1 regulate oxidative stress during erastin-induced ferroptosis in HepG2 hepatocellular carcinoma cells. Sci Rep. 2019; 9: 16185.

70. Wang Y, Chen H, Wei X. Circ 0007142 downregulates miR-874-3p-mediated GDPD5 on colorectal cancer cells. Eur J Clin Invest. 2021; 51: e135441.

71. Xian ZY, Hu B, Wang T, Cai JL, Zeng JY, Zou Q, et al. CircABCB10 silencing inhibits the cell ferroptosis and apoptosis by regulating the miR-326/CCL5 axis in rectal cancer. Neoplasma. 2020; 67: 1063-73.

72. Gai C, Liu C, Wu X, Yu M, Zheng J, Zhang W, et al. MT1DP loaded by folate-modified liposomes sensitizes erastin-induced ferroptosis via regulating miR-365a-3p/NRF2 axis in non-small cell lung cancer cells. Cell Death Dis. 2020; 11: 751.

73. Zhang H, Ge Z, Wang Z, Gao Y, Wang Y, Qu X. Circular RNA RHOT1 promotes progression and inhibits ferroptosis via mir-106a-5p/STAT3 axis in breast cancer. Aging. 2021; 13: 8115-26.

74. Bai T, Liang R, Zhu R, Wang W, Zhou L, Sun Y. MicroRNA-214-3p enhances erastin-induced ferroptosis by targeting ATF4 in hepatoma cells. J Cell Physiol. 2020; 235: 5637-48.

75. Gomaa A, Peng D, Chen Z, Soutto M, Abouelezz K, Corvalan A, et al. Epigenetic regulation of AURKA by miR-4715-3p in upper gastrointestinal cancers. Sci Rep. 2019; 9: 16970.

76. Zhang HY, Zhang BW, Zhang ZB, Deng QJ. Circular RNA TTBK2 regulates cell proliferation, invasion and ferroptosis via miR-761/ITGB8 axis in glioma. Eur Rev Med Pharmacol Sci. 2020; 24: 2585-2600.

77. Mao C, Wang X, Liu Y, Wang M, Yan B, Jiang Y, et al. A G3BP1-interacting lncRNA promotes ferroptosis and apoptosis in cancer via nuclear sequestration of p53. Cancer Res. 2018; 78: 3484-96.

78. Yang J, Cao XH, Luan KF, Huang YD. Circular RNA FNDC3B Protects Oral Squamous Cell Carcinoma Cells From Ferroptosis and Contributes to the Malignant Progression by Regulating miR-520d-5p/SLC7A11 Axis. Front Oncol. 2021; 11: 672724.

79. Wang Z, Chen X, Liu N, Shi Y, Liu Y, Ouyang L, et al. A Nuclear Long Non-Coding RNA LINC00618 Accelerates Ferroptosis in a Manner Dependent upon Apoptosis. Mol Ther. 2021; 29: 263-74.

80. Zhang H, Deng T, Liu R, Ning T, Yang H, Liu D, et al. CAF secreted miR-522 suppresses ferroptosis and promotes acquired chemo-resistance in gastric cancer. Mol Cancer. 2020; 19: 43.

81. Luo M, Wu L, Zhang K, Wang H, Zhang T, Gutierrez L, et al. miR-137 regulates ferroptosis by targeting glutamine transporter SLC1A5 in melanoma. Cell Death Differ. 2018; 25: 1457-72.

82. Wang M, Mao C, Ouyang L, Liu Y, Lai W, Liu N, et al. Long noncoding RNA LINC00336 inhibits ferroptosis in lung cancer by functioning as a competing endogenous RNA. Cell Death Differ. 2019; 26: 2329-43.

83. Tomita K, Fukumoto M, Itoh K, Kuwahara Y, Igarashi K, Nagasawa T, et al. MiR-7-5p is a key factor that controls radioresistance via intracellular $\mathrm{Fe}$ content in clinically relevant radioresistant cells. Biochem Biophys Res Commun. 2019; 518: 712-8.
84. Lyu N, Zeng Y, Kong Y, Chen Q, Deng H, Ou S, et al. Ferroptosis is involved in the progression of hepatocellular carcinoma through the circ0097009/miR-1261/SLC7A11 axis. Ann Transl Med. 2021; 9: 675.

85. Wu P, Li C, Ye DM, Yu K, Li Y, Tang H, et al. Circular RNA circEPSTI1 accelerates cervical cancer progression via miR-375/409-3P/515-5p-SLC7A11 axis. Aging. 2021; 13: 4663-73.

86. Lu X, Kang N, Ling X, Pan M, Du W, Gao S. MiR-27a-3p Promotes Non-Small Cell Lung Cancer Through SLC7A11-Mediated-Ferroptosis. Front Oncol. 2021; 11: 759346.

87. Yu Y, MohamedAl-Sharani H, Zhang B. EZH2-mediated SLC7A11 upregulation via miR-125b-5p represses ferroptosis of TSCC. Oral Dis. 2021; 00: 1-12.

88. Deng SH, Wu DM, Li L, Liu T, Zhang T, Li J, et al. miR-324-3p reverses cisplatin resistance by inducing GPX4-mediated ferroptosis in lung adenocarcinoma cell line A549. Biochem Biophys Res Commun. 2021; 549: 5460 .

89. Hou Y, Cai S, Yu S, Lin H. Metformin induces ferroptosis by targeting miR-324-3p/GPX4 axis in breast cancer. Acta Biochim Biophys Sin. 2021; 53: $333-41$.

90. He GN, Bao NR, Wang S, Xi M, Zhang TH, Chen FS. Ketamine induces ferroptosis of liver cancer cells by targeting lncRNA PVT1/miR-214-3p/GPX4. Drug Des Devel Ther. 2021; 15: 3965-78.

91. Xu Q, Zhou L, Yang G, Meng F, Wan Y, Wang L, et al. CircIL4R facilitates the tumorigenesis and inhibits ferroptosis in hepatocellular carcinoma by regulating the miR-541-3p/GPX4 axis. Cell Biol Int. 2020; 44: 2344-56.

92. Chen W, Fu J, Chen Y, Li Y, Ning L, Huang D, et al. Circular RNA circKIF4A facilitates the malignant progression and suppresses ferroptosis by sponging miR-1231 and upregulating GPX4 in papillary thyroid cancer. Aging. 2021; 13: 16500-12.

93. Yang W, Zhao J, Zhao Y, Li W, Zhao L, Ren Y, et al. Hsa_circ_0048179 attenuates free fatty acid-induced steatosis via hsa_circ_0048179/miR-188-3p/GPX4 signaling. Aging. 2020; 12: 23996-4008.

94. Niu Y, Zhang J, Tong Y, Li J, Liu B. Physcion 8-O- $\beta$-glucopyranoside induced ferroptosis via regulating miR-103a-3p/GLS2 axis in gastric cancer. Life Sci. 2019; 237: 116893.

95. Zhang K, Wu L, Zhang P, Luo M, Du J, Gao T, et al. miR-9 regulates ferroptosis by targeting glutamic-oxaloacetic transaminase GOT1 in melanoma. Mol Carcinog. 2018; 57: 1566-76.

96. Wu H, Liu A. Long non-coding RNA NEAT1 regulates ferroptosis sensitivity in non-small-cell lung cancer. J Int Med Res. 2021; 49: 300060521996183.

97. Ma L, Liang L, Zhou D, Wang S. Tumor suppressor miR-424-5p abrogates ferroptosis in ovarian cancer through targeting ACSL4. Neoplasma. 2021; 68: 165-173.

98. Yang X, Liu J, Wang C, Cheng KK yip, Xu H, Li Q, et al. miR-18a promotes glioblastoma development by down-regulating ALOXE3-mediated ferroptotic and anti-migration activities. Oncogenesis. 2021; 10: 15.

99. Sung H, Ferlay J, Siegel RL, Laversanne M, Soerjomataram I, Jemal A, et al. Global Cancer Statistics 2020: GLOBOCAN Estimates of Incidence and Mortality Worldwide for 36 Cancers in 185 Countries. CA Cancer J Clin. 2020; 70: 313.

100. Zappa C, Mousa SA. Non-small cell lung cancer: Current treatment and future advances. Transl Lung Cancer Res. 2016; 5: 288-300.

101. Ling Y, Zhang W, Wang P, Xie W, Yang W, Wang DA, et al. Three-dimensional (3D) hydrogel serves as a platform to identify potential markers of chondrocyte dedifferentiation by combining RNA sequencing. Bioact Mater. 2021; 6: 2914-26.

102. Hao XD, Liu YN, Hu SH, Pan XJ, Chen P. Association of macular corneal dystrophy with excessive cell senescence and apoptosis induced by the novel mutant CHST6. Exp Eye Res. 2021.

103. Yu H, Han Z, Xu Z, An C, Xu L, Xin H. RNA sequencing uncovers the key long non-coding RNAs and potential molecular mechanism contributing to XAV939-mediated inhibition of non-small cell lung cancer. Oncol Lett. 2019; 17: 4994-5004

104. Sui X, Hu N, Zhang Z, Wang Y, Wang P, Xiu G. ASMTL-AS1 impedes the malignant progression of lung adenocarcinoma by regulating SAT1 to promote ferroptosis. Pathol Int. 2021; 1-11.

105. Yao J, Chen X, Liu X, Li R, Zhou X, Qu Y. Characterization of a ferroptosis and iron-metabolism related lncRNA signature in lung adenocarcinoma. Cancer cell int. 2021; 21: 340 .

106. Fei X, Hu C, Wang X, Lu C, Chen H, Sun B, et al. Construction of a Ferroptosis-Related Long Non-coding RNA Prognostic Signature and Competing Endogenous RNA Network in Lung Adenocarcinoma. Front Cell Dev Biol. 2021; 9: 751490

107. Liu Y, Ao X, Yu W, Zhang Y, and Wang J. Biogenesis, functions, and clinical implications of circular RNAs in non-small cell lung cancer. Mol Ther Nucleic Acids. 2022; 27: 50-72

108. Wang S, Ma H, Fang J, Yu Y, Ren Y, Yu R. CircDTL Functions as an Oncogene and Regulates Both Apoptosis and Ferroptosis in Non-small Cell Lung Cancer Cells. Front Genet. 2021; 12: 743505

109. Zhu JH, De Mello RA, Yan QL, Wang JW, Chen Y, Ye QH, et al. MiR-139-5p/SLC7A11 inhibits the proliferation, invasion and metastasis of pancreatic carcinoma via PI3K/Akt signaling pathway. Biochim Biophys Acta Mol Basis Dis. 2020; 1866: 165747. 
110. Ni H, Qin H, Sun C, Liu Y, Ruan G, Guo Q, et al. MiR-375 reduces the stemness of gastric cancer cells through triggering ferroptosis. Stem Cell Res Ther. 2021; 12: 325.

111. Liu Y, Ao X, Ji G, Zhang Y, Yu W and Wang J. Mechanisms of Action And Clinical Implications of MicroRNAs in the Drug Resistance of Gastric Cancer. Front Oncol. 2021; 11: 768918.

112. Liu Y, Ding W, Yu W, Zhang Y, Ao X, Wang J. Long non-coding RNAs: Biogenesis, functions, and clinical significance in gastric cancer. Mol Ther Oncolytics. 2021; 23: 458-76.

113. Zhou Z, Lin Z, He Y, Pang X, Wang Y, Ponnusamy $M$, et al. The Long Noncoding RNA D63785 Regulates Chemotherapy Sensitivity in Human Gastric Cancer by Targeting miR-422a. Mol Ther Nucleic Acids. 2018;12: 40519.

114. Cai HJ, Zhuang ZC, Wu Y, Zhang YY, Liu X, Zhuang JF, et al. Development and validation of a ferroptosis-related lncRNAs prognosis signature in colon cancer. Bosn J Basic Med Sci. 2021.

115. Tang R, Wu Z, Rong Z, Xu J, Wang W, Zhang B, et al. Ferroptosis-related IncRNA pairs to predict the clinical outcome and molecular characteristics of pancreatic ductal adenocarcinoma. Brief Bioinform. 2021; 00: 1-13.

116. Li H, Liu L, Huang T, Jin M, Zheng Z, Zhang H, et al. Establishment of a novel ferroptosis-related lncRNA pair prognostic model in colon adenocarcinoma. Aging. 2021; 13.

117. Wei J, Zeng Y, Gao X, Liu T. A novel ferroptosis-related lncRNA signature for prognosis prediction in gastric cancer. BMC Cancer. 2021; 21: 1221.

118. Shan C, Zhang Y, Hao X, Gao J, Chen X, Wang K. Biogenesis, functions and clinical significance of circRNAs in gastric cancer. Mol Cancer. 2019; 18: 136.

119. Weng W, Wei Q, Toden S, Yoshida K, Nagasaka T, Fujiwara T, et al. Circular RNA ciRS-7-A promising prognostic biomarker and a potential therapeutic target in colorectal cancer. Clin Cancer Res. 2017; 23: 3918-28.

120. Bruix J, Reig M, Sherman M. Evidence-Based Diagnosis, Staging, and Treatment of Patients with Hepatocellular Carcinoma. Gastroenterology. 2016; 150: 835-53.

121. Knoops B, Clippe A, Bogard C, Arsalane K, Wattiez R, Hermans C, et al. Cloning and characterization of AOEB166, a novel mammalian antioxidant enzyme of the peroxiredoxin family. J Biol Chem. 1999; 274: 30451-8.

122. Liang J, Zhi Y, Deng W, Zhou W, Li X, Cai Z, et al. Development and validation of ferroptosis-related lncrnas signature for hepatocellular carcinoma. PeerJ. 2021; 9: e11627.

123. Nie Y, Li J, Wu W, Guo D, Lei X, Zhang T, et al. A Novel Nine-lncRNA Risk Signature Correlates With Immunotherapy in Hepatocellular Carcinoma. Front Oncol. 2021; 11: 706915.

124. Wang L, Ge X, Zhang Z, Ye Y, Zhou Z, Li M, et al. Identification of a Ferroptosis-Related Long Noncoding RNA Prognostic Signature and Its Predictive Ability to Immunotherapy in Hepatocellular Carcinoma. Front Genet. 2021; 12: 682082.

125. Xu Z, Peng B, Liang Q, Chen X, Cai Y, Zeng S, et al. Construction of a Ferroptosis-Related Nine-lncRNA Signature for Predicting Prognosis and Immune Response in Hepatocellular Carcinoma. Front Immunol. 2021; 12: 719175.

126. Liu Z, Wang $Q$, Wang $X, X u Z$, Wei $X$, Li J. Circular RNA cIARS regulates ferroptosis in HCC cells through interacting with RNA binding protein ALKBH5. Cell Death Discovery. 2020; 6: 72.

127. Yadav P, Sharma P, Sundaram S, Venkatraman G, Bera AK, Karunagaran D. SLC7A11/ xCT is a target of miR-5096 and its restoration partially rescues miR-5096-mediated ferroptosis and anti-tumor effects in human breast cancer cells. Cancer Lett. 2021; 522: 211-24.

128. Zhang K, Ping L, Du T, Liang G, Huang Y, Li Z, et al. A Ferroptosis-Related IncRNAs Signature Predicts Prognosis and Immune Microenvironment for Breast Cancer. Front Mol Biosci. 2021; 8: 678877.

129. Xu Z, Jiang S, Ma J, Tang D, Yan C, Fang K. Comprehensive Analysis of Ferroptosis-Related LncRNAs in Breast Cancer Patients Reveals Prognostic Value and Relationship With Tumor Immune Microenvironment. Front Surg. 2021; 8: 742360.

130. Bazhabayi M, Qiu X, Li X, Yang A, Wen W, Zhang X, et al. CircGFrA1 facilitates the malignant progression of HER-2-positive breast cancer via acting as a sponge of miR-1228 and enhancing AIFM2 expression. J Cell Mol Med. 2021; 25: 10248-56.

131. Martens-Uzunova ES, Böttcher R, Croce CM, Jenster G, Visakorpi T, Calin GA. Long noncoding RNA in prostate, bladder, and kidney cancer. Eur Urol. 2014; 65: $1140-51$.

132. Luo W, Wang J, Xu W, Ma C, Wan F, Huang Y, et al. LncRNA RP11-89 facilitates tumorigenesis and ferroptosis resistance through PROM2-activated iron export by sponging miR-129-5p in bladder cancer. Cell Death Dis. 2021;12: 1043

133. Xing XL, Yao ZY, Ou J, Xing C, Li F. Development and validation of ferroptosis-related lncRNAs prognosis signatures in kidney renal clear cell carcinoma. Cancer Cell Int. 2021; 21: 591

134. Deutsch E, Maggiorella L, Eschwege P, Bourhis J, Soria JC, Abdulkarim B. Environmental, genetic, and molecular features of prostate cancer. Lancet Oncol. 2004; 5: 303-13.

135. Zhang Y, Guo S, Wang S, Li X, Hou D, Li H, et al. LncRNA OIP5-AS1 inhibits ferroptosis in prostate cancer with long-term cadmium exposure through miR-128-3p/SLC7A11 signaling. Ecotoxicol Environ Saf. 2021; 220: 112376.
136. Berindan-Neagoe I, Monroig P del C, Pasculli B, Calin GA. MicroRNAome genome: A treasure for cancer diagnosis and therapy. CA Cancer J Clin. 2014; 64: 311-36.

137. He L, Zhu W, Chen Q, Yuan Y, Wang Y, Wang J, et al. Ovarian cancer cell-secreted exosomal miR-205 promotes metastasis by inducing angiogenesis. Theranostics. 2019; 9: 8206-20.

138. Shi J, Fu H, Jia Z, He K, Fu L, Wang W. High Expression of CPT1A Predicts Adverse Outcomes: A Potential Therapeutic Target for Acute Myeloid Leukemia. EBioMedicine. 2016; 14: 55-64.

139. Khan G, Orchard K, Guinn B. Antigenic Targets for the Immunotherapy of Acute Myeloid Leukaemia. J Clin Med. 2019; 8: 134

140. Bester AC, Lee JD, Chavez A, Lee YR, Nachmani D, Vora S, et al. An Integrated Genome-wide CRISPRa Approach to Functionalize IncRNAs in Drug Resistance. Cell. 2018; 173: 649-664.e20.

141. Louis DN, Ohgaki H, Wiestler OD, Cavenee WK, Burger PC, Jouvet A, et al. The 2007 WHO classification of tumours of the central nervous system. Acta Neuropathol. 2007; 114: 97-109.

142. Liu Y. Targeting the non-canonical AKT-FOXO3a axis: A potential therapeutic strategy for oral squamous cell carcinoma. EBioMedicine. 2019; 49: 6-8.

143. Tang Y, Li C, Zhang YJ, Wu ZH. Ferroptosis-related long non-coding RNA signature predicts the prognosis of head and neck squamous cell carcinoma. Int J Biol Sci. 2021; 17: 702-11.

144. Wang HH, Ma JN, Zhan XR. Circular RNA Circ_0067934 Attenuates Ferroptosis of Thyroid Cancer Cells by miR-545-3p/SLC7A11 Signaling. Front Endocrinol. 2021; 12: 670031.

145. Xue CC, Li MH, Zhao Y, Zhou J, Hu Y, Cai KY, et al. Tumor microenvironment-activatable Fe-doxorubicin preloaded amorphous $\mathrm{CaCO} 3$ nanoformulation triggers ferroptosis in target tumor cells. Sci Adv. 2020; 6: eaax1346.

146. Yao X, Yang P, Jin Z, Jiang Q, Guo R, Xie R, et al. Multifunctional nanoplatform for photoacoustic imaging-guided combined therapy enhanced by CO induced ferroptosis. Biomaterials. 2019; 197: 268-83. 